University of Louisville

ThinkIR: The University of Louisville's Institutional Repository

\title{
The effect of interspecies signaling on Stenotrophomonas maltophilia growth.
}

Zachary Ethan Dickinson

Follow this and additional works at: https://ir.library.louisville.edu/honors

Part of the Bacterial Infections and Mycoses Commons, and the Biology Commons

\section{Recommended Citation}

Dickinson, Zachary Ethan, "The effect of interspecies signaling on Stenotrophomonas maltophilia growth." (2016). College of Arts \& Sciences Senior Honors Theses. Paper 111.

http://doi.org/10.18297/honors/111

This Senior Honors Thesis is brought to you for free and open access by the College of Arts \& Sciences at ThinkIR: The University of Louisville's Institutional Repository. It has been accepted for inclusion in College of Arts \& Sciences Senior Honors Theses by an authorized administrator of ThinkIR: The University of Louisville's Institutional Repository. This title appears here courtesy of the author, who has retained all other copyrights. For more information, please contact thinkir@louisville.edu. 
The Effect of Interspecies Signaling on

Stenotrophomonas maltophilia Growth

By

Zachary Ethan Dickinson

Submitted in partial fulfillment of the requirements

for Graduation Summa Cum Laude

and

for Graduation with Honors from the Department of Biology

University of Louisville

May 2016 



\begin{abstract}
The Effect of Interspecies Signaling on Stenotrophomonas maltophilia Growth Zachary Dickinson
\end{abstract}

May 2016

Cystic fibrosis (CF) is a genetic disorder that effects over 70,000 people worldwide, and is particularly prevalent in those of Caucasian descent. CF increases susceptibility to chronic infections of the lungs and GI tract, often leading to decreased quality of life and early death to afflicted individuals. No cure is available, and so treatment is limited to treating the frequent infections that afflict CF patients. Several species of bacteria are commonly implicated in these infections, including Stenotrophomonas maltophilia. Infections of this species is of particular interest as it has been shown to be highly antibiotic resistant. This is a concern when it is part of coinfections with other, more virulent species, such as Pseudomonas aeruginosa. It has been shown that these two species are much more difficult to treat when they are present together than either one is alone.

There is thought to be a molecular signaling mechanism through which these two species are able to communicate and increase each other's resilience, although the exact identity of that mechanism is unknown. This study began by identifying (based on bioinformatics) six likely candidates for quorum sensing receptor proteins. Through insertional interruption, five of these genes were made nonfunctional and these constructs were tested to identify a growth phenotype of $S$. maltophilia in the presence and absence of $P$. aeruginosa. Results of this study may suggest that none of the selected gene products play a role in communication between these two species, but additional assays may identify a connection. 


\section{Table of Contents}

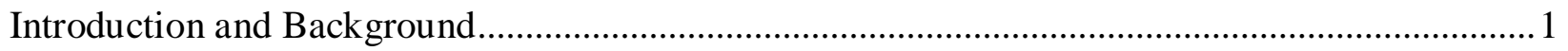

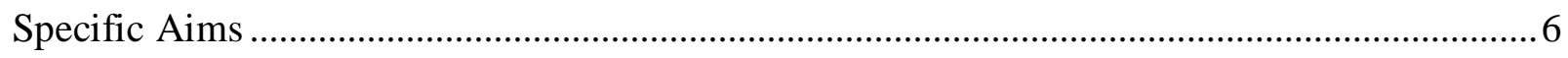

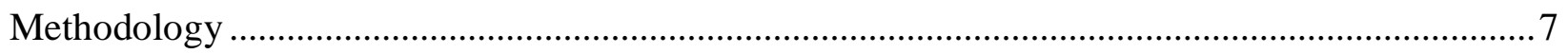

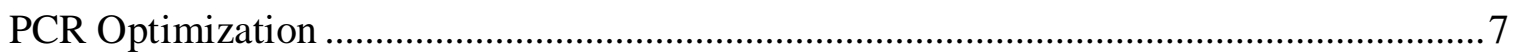

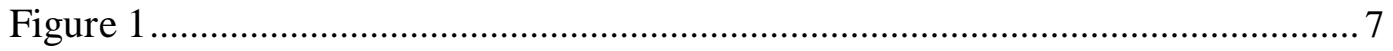

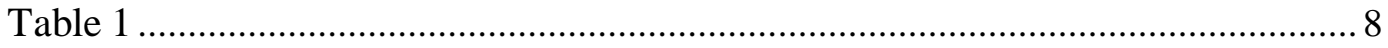

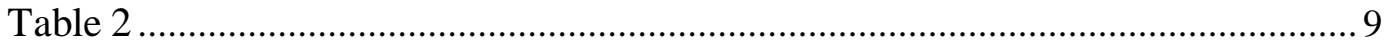

Purification of pEX18Tc............................................................................................. 11

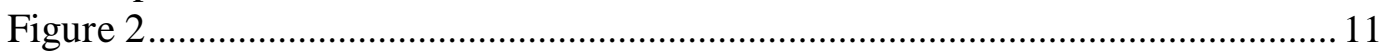

Purifications and Restriction Enzyme Digest ..................................................................... 12

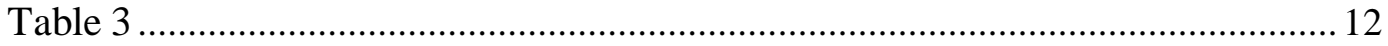

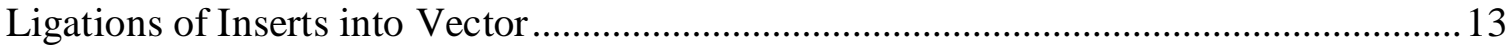

Preparation of competent E. coli DH5 $\alpha$ cells ......................................................................13

Transformation of ligations into chemically competent $E$. coli .......................................14

Conjugation from E. coli into S. maltophilia ……………............................................... 15

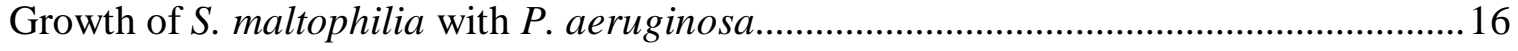

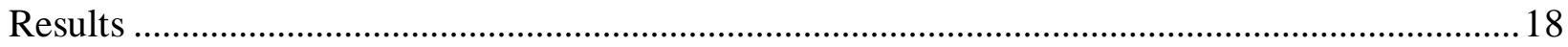

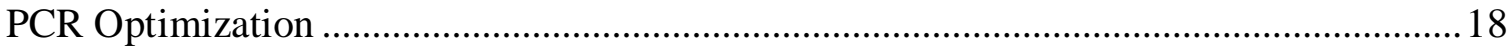

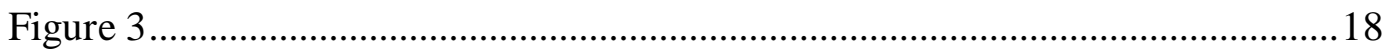

Figure 4 ............................................................................................... 19

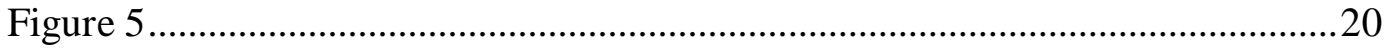

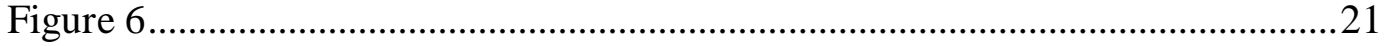

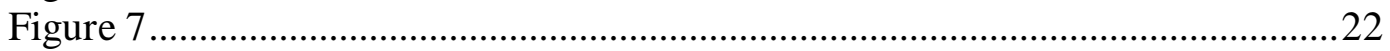

Purifications, Restriction Enzyme Digestion and Ligations...............................................23

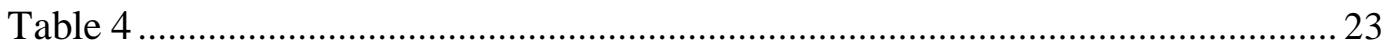

Transformation of ligations into chemically competent $E$. coli ........................................23

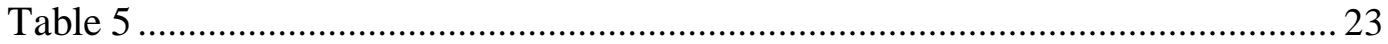

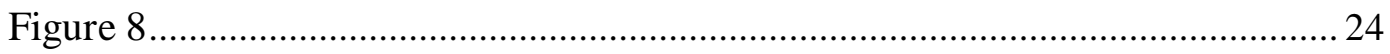

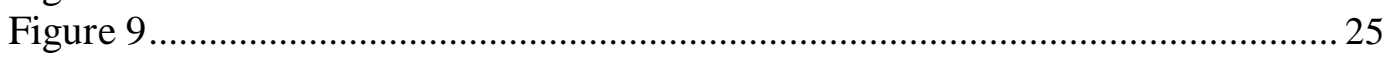

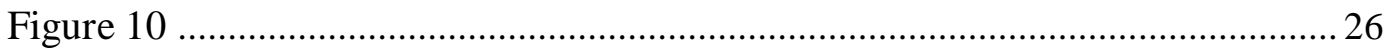

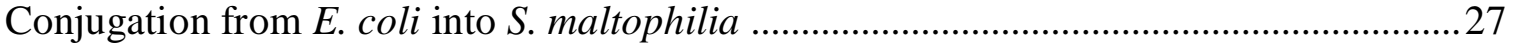

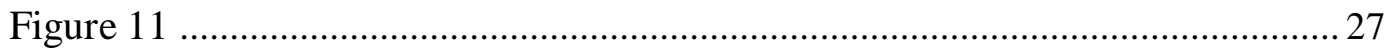

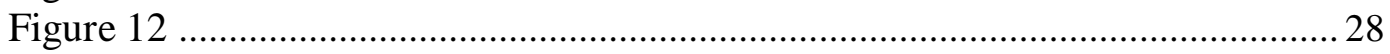

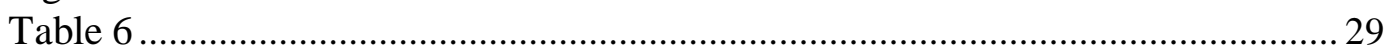

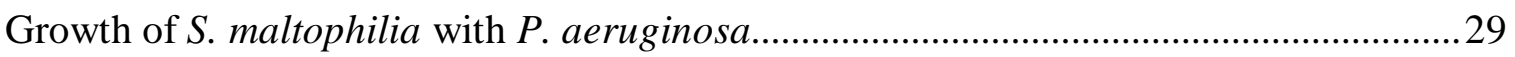

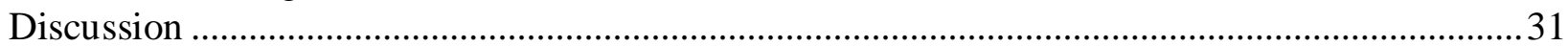

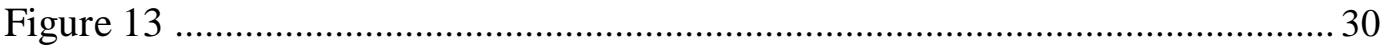

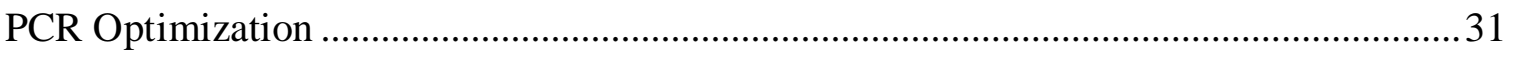

Transformation of ligations into chemically competent $E$. coli ...........................................31

Conjugation and Growth Testing ...................................................................................... 32

References 


\section{Introduction and Background:}

Stenotrophomonas maltophilia is a species of gram-negative, nonfermentive bacteria that is not generally pathogenic to healthy humans ${ }^{(2)}$. S. maltophilia is able to colonize a large variety of environmental habitats including soils, fluid media, and the surfaces of plants ${ }^{(3)}$. It general, $S$. maltophilia is unable to infect human hosts because it is unable to easily bypass the basic immune defenses ${ }^{(3)}$. In spite of this, S. maltophilia has been gaining increased attention as an emerging pathogen in the past two decades for a number of reasons, especially in hospital settings $^{(2,6,8,10)}$. S. maltophilia has been shown to be highly resistant to a suite of antibiotics, including quinones, $\beta$-lactams (carbapenems, penicillins, cephalosporins) and aminoglycosides $^{(3)}$. Additionally, S. maltophilia is able to colonize plastic surfaces (as a result of adaptations that include a positively charged surface and fimbrial adhesions). As a result, it is now commonly found on a variety of common medical equipment, including catheters, endotracheal tubes, and other indwelling-lines that aid in the bacteria's transmission into its host $^{(3)}$. Once entry into the a host has been established, S. maltophilia infection primarily results in pneumonia, but bloodstream infections (septicemia), infection on the skin and soft tissues, urinary tract infections, intraabdominal infections, and even ocular infections can also occur ${ }^{(8)}$.

S. maltophilia infections occur most frequently in immunocompromised and chronically ill patients ${ }^{(2)}$ and are of particular concern for patients with cystic fibrosis $(\mathrm{CF})$. Cystic fibrosis is the most prevalent genetic disorder among people of European descent ${ }^{(9)}$. As a genetic disorder, cystic fibrosis is a result of mutations in the gene encoding the Cystic Fibrosis Transmembrane Conductance Regulator (CFTR) ${ }^{(1)}$. Most often (in about $71 \%$ of patients with CF), the dysfunction is caused by a deletion of the phenylalanine codon 508 in the CTFR gene ${ }^{(9)}$. CTFR functions as a chloride transporter and is a critical component of maintaining proper 
chemiosmotic balances across cellular membranes ${ }^{(9)}$. As such, this protein is critical in the production of sweat, digestive fluids, and mucus. Individuals born with a faulty copy of CTFR from each parent are unable to produce normal secretions.

The inability to maintain chemiosmotic balance is especially problematic for the secretions in the lungs. In healthy individuals, the lungs secrete a thin lubricant. Any foreign material, such as dust or potential pathogens, is caught in this secretions and the cilia lining the epithelia of the lungs can easily clear this secretion, bringing it to the back of the throat where it can be coughed up or swallowed. Since CF patients do not maintain the proper balance of extracellular ions, the volume of pericellular fluid is drastically reduced and mucus clearance is severely hindered ${ }^{(1)}$. This creates a warm, humid environment that is created is ideal for colonization by bacteria, setting up CF patients for chronic infections in the lungs ${ }^{(1)}$.

Historically, CF was a deadly condition that resulted in severely shortened life expectancies of afflicted individuals ${ }^{(7)}$. This was due primarily to chronic infections of the respiratory tract that would eventually cause a severe decline in lung function and lung failure. This process begins as an inflammatory response to the growing populations of bacteria in the lungs. The body attempts to mount a defense, leading to a chronic neutrophil-dominated infection and persistent inflammatory response resulting in tissue necrosis and tissue scarring that prevents gas exchange in respiration ${ }^{(7)}$. This decline continues until the patient receives a transplant of a healthy lung or lung function degrades sufficiently enough as to cause death. In recent decades, thanks to the advent of advancing aggressive antibiotic therapies, individuals with CF are living well beyond their forties, whereas before they often did not survive childhood. Even with antimicrobial therapies, the main problem for CF patients is the chronic inflammatory state that results from persistent infections. With preemptive and aggressive antibiotic treatment, 
these chronic infections take longer to develop, and the resultant tissue necrosis occurs much later in life. As a result, quality of life for CF patients is greatly improved and life expectancy has increased by decades ${ }^{(7)}$.

As CF patients are increasingly able to live well into adulthood, the complications that arise from persistent antibiotic use are becoming clearer. As CF patients age, they begin to acquire bacteria not so easily cleared by many front-line antibiotics. These bacteria include Stenotrophomonas maltophilia, Pseudomonas aeruginosa, Staphylococcus aureus, Haemophilus influenzae, Burkholderia cepacia, Achromobacter xylosoxidans, and Prevotella intermedia(1). The most concerning of these is also the most common: Pseudomonas aeruginosa. In adults, $P$. aeruginosa will colonize various portions of the conducting and respiratory zones of the respiratory tract, and if not treated can lead to the chronic infection state described above ${ }^{(1)}$. The presence of multi-antibiotic resistant $P$. aeruginosa is a marker of advanced disease requiring extensive antibiotic therapy ${ }^{(1)}$. However, treatment for this bacteria exists in the form of aggressive mixes of intravenous, oral, and inhaled antibiotics ${ }^{(1)}$. If caught and treated early enough, the infection can be kept from developing into a serious, life-threatening state.

Current research involves attempts to identify more effective treatments for the advanced stages of chronic bacterial infections. These studies have shown that the presence of biofilms increases the complexity of treating such infections. Biofilms are a community of microbial organisms (either one or multiple species of bacteria) that inhabit a favorable growing environment such as an extracellular mucus (in the case of biofilms for cystic fibrosis patients) but also including nonorganic environments such as rocks ${ }^{(3)}$. The exact mechanism that facilitates biofilm creation and adhesion is not well understood for most species ${ }^{(5)}$. However, it is understood that the physical structure of the biofilm (which often exists as an extracellular matrix 
or slime layer secreted by the organisms) creates an environment that is both ideal for bacterial growth and adaptation while simultaneously severely hindering the effectiveness of antibiotic treatment ${ }^{(3)}$. As an exacerbating factor, these biofilms have been shown to be ideal habitats promoting colonization by multiple species of bacteria, including many of those listed above ${ }^{(1)}$.

Participation in biofilm communities by S. maltophilia is particularly problematic. Likely thanks to its origin in soil and aquatic ecosystems, $S$. maltophilia contains a diverse set of adaptations suited to hostile environments. These adaptations include an intrinsic high level of resistance to multiple antibiotics, the ability to utilize horizontal gene transfer, and a suite of efflux pumps that constitutively pump out harmful chemicals ${ }^{(11)}$. Although S. maltophilia is not extraordinarily virulent, the frequency of isolation of S. maltophilia from chronically ill patients and compromised patients (including CF patients) is on the rise thanks in part due to improved detection methods ${ }^{(2)}$ and more effective antibiotic treatment. Consequently, its natural resistance has become a concern. S. maltophilia could potentially act as a reservoir for resistance that could be passed on to more pathogenic organisms, such as $P$. aeruginosa ${ }^{(11)}$.

As the role of $S$. maltophilia in chronic infections emerges, the rates of its coinfections with $P$. aeruginosa and their possible synergism becomes an important topic of study. A study on biofilm formation between $P$. aeruginosa and S. maltophilia on in vitro IB3-1 cells (lung epithelial tissue) showed that, once a biofilm is created by P. aeruginosa, S. maltophilia is more readily able to adhere and colonize that biofilm ${ }^{(4)}$. A biofilm containing multiple species is of concern, as it imposes greater challenges to conventional treatments ${ }^{(7)}$, although again the exact etiology of this challenge has not been well understood. Furthermore, for CF patients in particular there is concern about coinfections, as the presence of S. maltophilia has been shown 
to disrupt ion concentrations, which would further exacerbate the chemiosmotic problems resulting from the faulty $\mathrm{CFTR}^{(6)}$.

Although the specific nature of the effectors resulting in synergism between bacteria that makes the multi-species biofilms so robust are not fully understood, intercellular signaling is expected to be involved ${ }^{(5,7)}$. Quorum sensing could play a role, as it is known to play an important role in the virulence of $P$. aeruginosa ${ }^{(14)}$ P. aeruginosa uses three different types of signaling systems (for quorum sensing) to communicate with itself as well as other species. The first two use acylated homoserine lactones (AHLs) that use two different receptor proteins: Las and $\mathrm{Rhl}^{(16)}$. A third signaling system uses molecules belonging to the 2-alkyl-4-quinolone family (often referred to as PQS, or Pseudomonas quinolone signal) and is known to bind a response effector (pqsE) $)^{(15,16)}$. Additionally, various strains of $P$. aeruginosa have been shown to secrete a suite of other compounds that may have signaling capabilities including pyocyanins, which, while they have inhibitory effects at high concentrations, have been shown to modulate gene regulation in lower concentrations ${ }^{(16)}$. As a result, this species is able to sense and respond appropriately to its own cell density as well as the composition of other species in its environment. Since $S$. maltophilia is a member of the $\gamma$-proteobacteria like $P$. aeruginosa and is likely responding to signals produced by $P$. aeruginosa, it is highly probable that it uses receptors similar to those produced by $P$. aeruginosa. Additionally, $S$. maltophilia is capable of horizontal gene transfer, which could possibly play a role in increasing the resistance of adjacent species $^{(7)}$. Ultimately, there must be a mechanism behind the synergistic behavior of these species, although the area is understudied ${ }^{(7)}$. 


\section{Specific Aims}

Initial computer genome mapping of S. maltophilia's genome of the K279a strain has identified two genes, $r p f G$ and Smlt_0278, as encoding quorum sensing proteins ${ }^{(11)}$. Further bioinformatics analysis identified Smlt_0184,Smlt_1432,Smlt_1839, and Smlt_3567 as additional strong candidates to encode other quorum sensing proteins through their high similarity to known receptors. The purpose of this study was to determine, through insertional interruption (using the plasmid vector pEX18Tc), if these genes have any significant effect on the growth of $S$. maltophilia both with and without the presence of $P$. aeruginosa. 


\section{Methodology:}

\section{PCR Optimization}

Primers binding to internal sites in each of the six genes were purchased from Integrated DNA Technologies (Coralville, Iowa). These primers were designed to amplify an internal $\sim 500$ bp fragment of each gene. This fragment, when cloned into pEX18Tc (which is not expected to

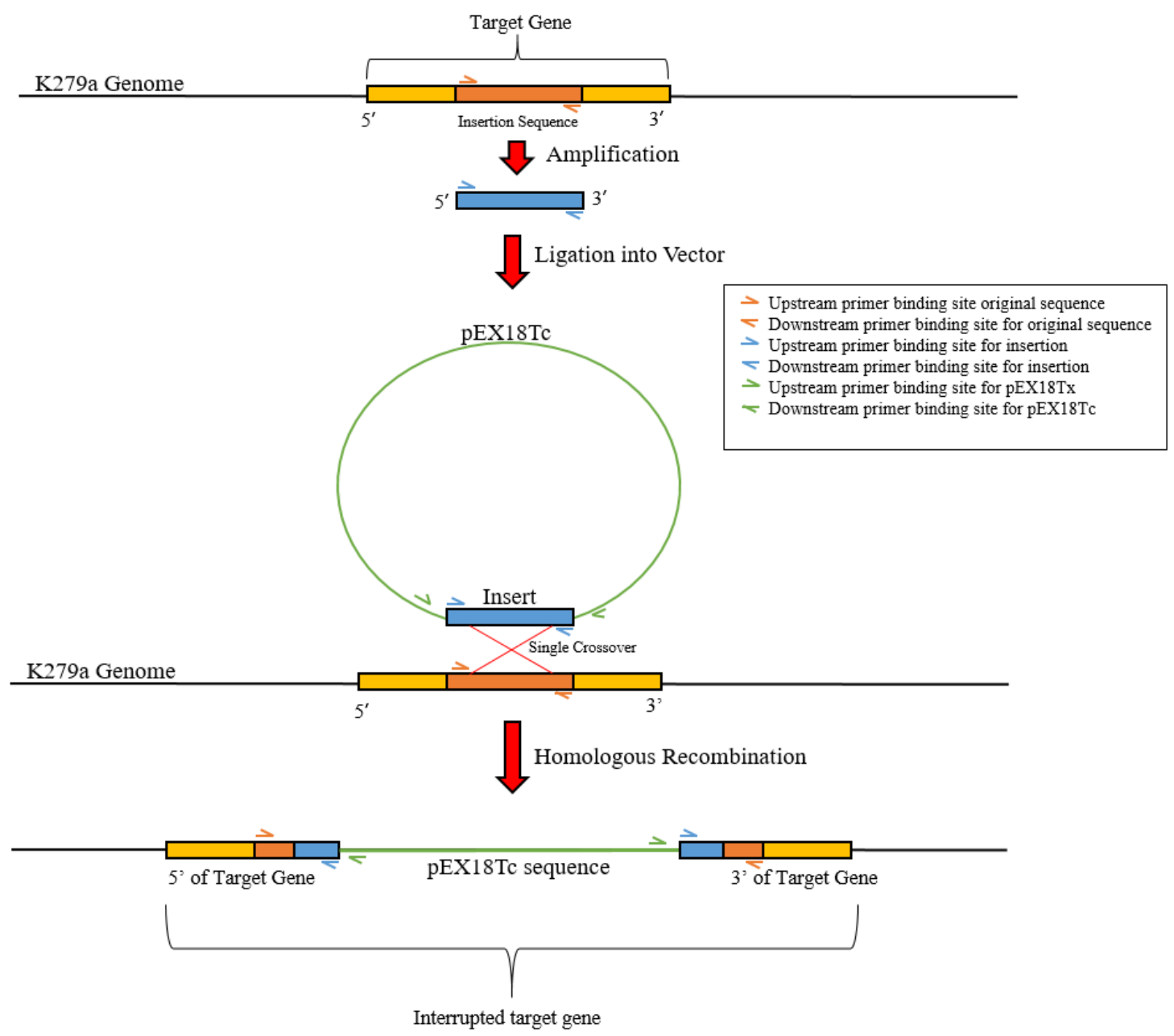

Figure 1: Summary of amplification, ligation, and insertion events.

be able to replicate in $\mathrm{K} 279 \mathrm{a}^{(13)}$ ) would result in interruption of the desired gene due to single crossover of the plasmid into the genome. This would occur because the internal fragment 
contains neither the start nor the stop of the gene, so neither gene fragment resulting from the recombination would result in a functional protein (Figure 1).

The primers were based upon the genome sequence of the S. maltophilia strain K279a, in which the transmembrane receptors were identified ${ }^{(11)}$. A summary of the genes, including the expected size of the product and the sequence of the primers, is provided in Table 1.

\begin{tabular}{|c|c|c|c|}
\hline Gene & $\begin{array}{l}\text { Expected Product } \\
\text { Size (bp) }\end{array}$ & Reverse Primer & Forward Primer \\
\hline$r p f G$ & 645 & $\begin{array}{l}5^{\prime}-\text { GCG GGA TCC CCT GGT } \\
\text { GCG AGG CGC ACC C-3' }\end{array}$ & $\begin{array}{l}5^{\prime}-\text { GCG GAA TTC CGC } \\
\text { GCT TCC AGC GGG ATC G- } \\
3^{\prime}\end{array}$ \\
\hline Smlt_0184 & 366 & $\begin{array}{l}5^{\prime}-\text { GCG GGA TCC TAA GGC } \\
\text { GGC GAC GCC GAC GGC-3' }\end{array}$ & $\begin{array}{l}5^{\prime}-\text { GCG GAA TTC TTA } \\
\text { CAC GGC CAT AGA ACT } \\
\text { CGC CG- }-3^{\prime}\end{array}$ \\
\hline Smlt_0278 & 628 & $\begin{array}{l}5^{\prime} \text {-GCG GGA TCC TAA GGA } \\
\text { AGA CTC GCG CGCC GCT-3 } \\
,\end{array}$ & $\begin{array}{l}5^{\prime}-\text { GCG GAA TTC TTA } \\
\text { GCC AAG CAG TTC CTG } \\
\text { CAG ATC-3' }\end{array}$ \\
\hline Smlt_1432 & 421 & $\begin{array}{l}5^{\prime}-\text { GCG GGA TCC CCA } \\
\text { TCG CGG CAC GGT AGG G- } \\
3^{\prime}\end{array}$ & $\begin{array}{l}5^{\prime}-\text { GCG GAA TTC CGC } \\
\text { CAC CAT TGC GAC CGC G - } \\
3^{\prime}\end{array}$ \\
\hline Smlt_1839 & 567 & $\begin{array}{l}5^{\prime} \quad \text { - GCG GGA TCC GTT } \\
\text { CTC CAC CGT GCG CGT GG - } \\
3^{\prime}\end{array}$ & $\begin{array}{l}5^{\prime}-\text { GCG GAA TTC GGT } \\
\text { CCT GCG AGG CAT CGC G- } \\
3^{\prime}\end{array}$ \\
\hline Smlt_3567 & 715 & $\begin{array}{l}\text { 5' GCG GGA TCC TAA GCG } \\
\text { GCA GAC TGC GGT GAT GG } \\
-3^{\prime}\end{array}$ & $\begin{array}{l}5^{\prime} \text { - GCG GAA TTC TTA } \\
\text { GCC GAT CGC GGC CTG } \\
\text { CAC G -3' }\end{array}$ \\
\hline
\end{tabular}

Multiple PCR conditions were tested in order to determine those resulting in the best amplification of the desired products. To this effect, the polymerases Phusion (New England Biolabs(NEB), Ipswich, MA), HotStar (Qiagen, Hilden, Germany), and GoTaq green (Promega, Madison, Wisconsin) were used. Gel electrophoresis was performed after each trial. A $2 \log$ DNA ladder (NEB) was used as size comparison in order to determine if desired gene products were amplified correctly. Table 2 outlines the conditions that were used. 


\begin{tabular}{|l|l|l|}
\hline \multicolumn{3}{|c|}{ Table 2: Basic PCR cycling used for the majority of PCR reactions. } \\
\hline Step \# & Temp (Degrees Celsius) & Time (min:sec) \\
\hline 1 & 95 & $5: 00$ \\
\hline 2 & 95 & $0: 30$ \\
\hline 3 & 55 & $1: 00$ \\
\hline 4 & 72 & $2: 00$ \\
\hline 5 & Go to step two 35 times. \\
\hline 6 & 72 & $10: 00$ \\
\hline 7 & 4 & hold \\
\hline
\end{tabular}

\section{$\underline{\text { GoTaq Green }}$}

PCR mixes with GoTaq green were made with $5 \mu$ l of GoTaq Green mix, $1 \mu l$ each of the desired product's $20 \mathrm{pM}$ forward and reverse primer, $1 \mu 1$ approximately $200 \mathrm{ng} / \mu \mathrm{l}$ phenol/chloroform-purified K279a genomic DNA that had been previously obtained by other researchers, and $2 \mu \mathrm{l}$ of water to bring the entire solution to $10 \mu$ l.

\section{$\underline{\text { HotStar }}$}

Each PCR mix prepared with HotStar as a polymerase were comprised of $1 \mu 1$ of HotStar polymerase, $10 \mu 1$ of HotStar $5 \times$ Buffer, and $10 \mu 1$ of Q buffer. To this, $1 \mu 1$ each of the $20 \mathrm{pM}$ forward and reverse primer of desired amplification product was added. $1 \mu 1 \mathrm{of} \sim 200 \mathrm{ng} / \mu \mathrm{l}$ K279a genomic DNA was added and $24 \mu 1$ of water to bring the entire solution to $50 \mu \mathrm{L}$.

\section{$\underline{\text { Phusion }}$}

Phusion PCR mixes were comprised of $0.5 \mu 1$ of Phusion, $10 \mu 1$ of Phusion $5 \times$ buffer Hi GC, and $10 \mu \mathrm{l}(10 \mathrm{mM}) \mathrm{dNTPs}$. To this, $1 \mu \mathrm{l}$ each of the $20 \mathrm{pM}$ forward and reverse primer of desired amplification product was added. $1 \mu 1$ of $\sim 200 \mathrm{ng} / \mu 1$ phenol/chloroform-purified K279a 
genomic DNA was added and $35.5 \mu \mathrm{l}$ of water to bring the entire solution to $50 \mu \mathrm{l}$. The extension temperature (step 4, table 2) was adjusted to $68^{\circ} \mathrm{C}$ for Phusion.

\section{$\underline{\text { Gel Electrophoresis }}$}

To determine whether PCR products contained products of the appropriate size, the products were examined by electrophoresis on $1 \%$ agarose gels that had been stained with ethidium bromide. Gels were loaded with $5 \mu$ l of the desired PCR mix (with loading dye added as necessary) or 2-log DNA ladder and then run at constant 100 volts. Duration varied between 30-60 minutes, depending on the size of the gel. The image of each gel was obtained after exposure to ultraviolet light in a VersaDoc Model 4000 (Bio-Rad, Hercules, CA). PCR products were compared to the 2 Log DNA ladder for size determination.

\section{Modifications to basic PCR procedure}

In many cases, extra bands indicating nonspecific amplification were observed. To rectify this, the desired bands were gel purified using the QIAquick Gel Extraction Kit (Qiagen, Hilden, Germany), and this purification product was used in a second round of amplification using HotStar polymerase as described above. This methodology was only performed with HotStar polymerase, since GoTaq green was only used for screening (the products never needed to be isolated) and Phusion was ineffective for specific amplification of the desired products.

Gel extraction followed the manufacturer protocol, and began with excision of the DNA fragment from the gel with a scalpel. The gel slice was weighed, and 3 volumes of Buffer QG was added for 1 volume gel (100 mg gel was estimated to be roughly equivalent to $100 \mu \mathrm{l}$ as per kit instructions). This was incubated at $50^{\circ} \mathrm{C}$ for 10 minutes or until the gel dissolved completely. To this 1 gel volume of isopropanol was added. This mixture was placed in the QIAquick Spin Columns and centrifuged at 14,000 rpm in a tabletop centrifuge for 1 minute. The flowthrough 
was discarded. $500 \mu 1$ of Buffer QG was added to this column and centrifuged again for 1 minute at 14,000 rpm. The flowthrough was discarded again. $750 \mu 1$ of Buffer PE was added, and centrifugation was repeated. Again, flow through was discarded. The column was then transferred to a new microcentrifuge tube. To elute the DNA, $50 \mu 1$ of $\mathrm{ddH}_{2} \mathrm{O}$ was added and centrifugation was repeated. The resulting mixture could be used again in subsequent PCR reactions to amplify desired products.

\section{Purification of pEX18Tc}

The vector into which the PCR-amplified gene products were to be inserted for interrupting the genes in $S$. maltophilia, pEX18Tc, is able to replicate in E. coli but not $S$. maltophilia and provides tetracycline resistance ${ }^{(13)}$. In order to have sufficient quantities of pEX18Tc for cloning, it was first purified from DH5 $\alpha$ E. coli using the Qiagen Spin Miniprep kit using the manufacturer's protocol (Qiagen, Hilden, Germany). The purified pEX18Tc was quantified on a Nanodrop 2000 (Nanodrop, Wilmington, Delaware) by measuring absorbance at $260 \mathrm{~nm}$.

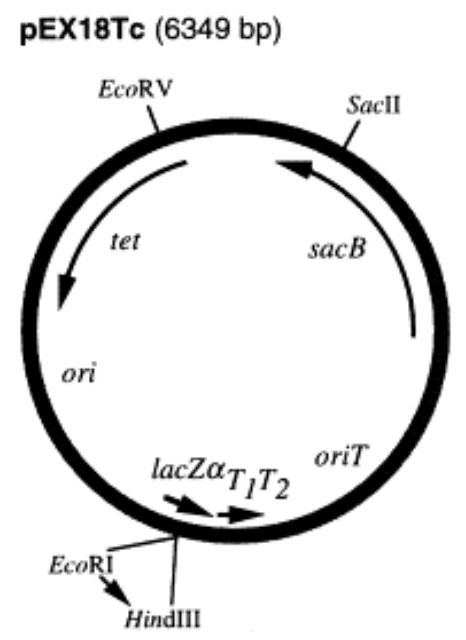

Figure 2: Structure of the plasmid pEX18Tc used as a vector ${ }^{(13)}$. 


\section{Purifications and Restriction Enzyme Digestion}

Once amplification of the desired PCR products had been confirmed with gel

electrophoresis, the DNA products and plasmid into which they were going to be inserted were digested using restriction enzymes BamHI and EcoRI (NEB), both of which have unique restriction sites in the multicloning locus that interrupts $l a c Z \alpha$ in $\mathrm{pEX} 18 \mathrm{Tc}$ (Figure 2). Prior to digestion, the amplification products were purified from the PCR mixes. This was done using QIAquick PCR Purification Kit (Qiagen, Hilden, Germany) according to manufacturer instructions.

The resulting mixture was analyzed on a Nanodrop 2000 (using $\mathrm{ddH}_{2} \mathrm{O}$ as a blank) to determine the concentration of purified gene products so that $1 \mu \mathrm{g}$ of each of the amplification products and $6 \mu \mathrm{g}$ of pEX18Tc could be digested in the mixtures outlined in Table 3.

\begin{tabular}{|c|c|c|c|c|c|c|c|}
\hline Digest & $\begin{array}{l}\text { 1: } \\
r p f G\end{array}$ & $\begin{array}{l}\text { 2: } \\
\text { Smlt_0184 }\end{array}$ & $\begin{array}{l}\text { 3: } \\
\text { Smlt } 0278\end{array}$ & $\begin{array}{l}4: \\
\text { Smlt_1432 }\end{array}$ & $\begin{array}{l}\text { 5: } \\
\text { Smlt } 1839\end{array}$ & $\begin{array}{l}\text { 6: } \\
\text { Smlt } 3567\end{array}$ & $\begin{array}{l}\text { 7: } \\
\text { pEX18TC }\end{array}$ \\
\hline $\begin{array}{l}10 \times \\
\text { CutSmart } \\
\text { Buffer }\end{array}$ & $2 \mu \mathrm{l}$ & $2 \mu \mathrm{l}$ & $2 \mu 1$ & $2 \mu \mathrm{l}$ & $2 \mu 1$ & $2 \mu \mathrm{l}$ & $4 \mu \mathrm{l}$ \\
\hline BamHI & $1 \mu \mathrm{l}$ & $1 \mu \mathrm{l}$ & $1 \mu 1$ & $1 \mu \mathrm{l}$ & $1 \mu \mathrm{l}$ & $1 \mu \mathrm{l}$ & $6 \mu 1$ \\
\hline EcoRI & $1 \mu l$ & $1 \mu \mathrm{l}$ & $1 \mu \mathrm{l}$ & $1 \mu \mathrm{l}$ & $1 \mu \mathrm{l}$ & $1 \mu l$ & $6 \mu 1$ \\
\hline CIP & 0 & 0 & 0 & 0 & 0 & 0 & $1 \mu \mathrm{l}$ \\
\hline $\begin{array}{l}\text { DNA to } \\
\text { be } \\
\text { Digested }\end{array}$ & $6.3 \mu \mathrm{l}$ & $6.5 \mu \mathrm{l}$ & $9.0 \mu \mathrm{l}$ & $9.9 \mu \mathrm{l}$ & $8.7 \mu \mathrm{l}$ & $7.4 \mu 1$ & $18.3 \mu \mathrm{l}$ \\
\hline $\mathrm{ddH}_{2} \mathrm{O}$ & $9.7 \mu \mathrm{l}$ & $9.5 \mu l$ & $7.0 \mu \mathrm{l}$ & $6.1 \mu \mathrm{l}$ & $7.3 \mu \mathrm{l}$ & $8.6 \mu \mathrm{l}$ & $4.7 \mu l$ \\
\hline $\begin{array}{l}\text { Total } \\
\text { volume }\end{array}$ & $20 \mu \mathrm{l}$ & $20 \mu \mathrm{l}$ & $20 \mu \mathrm{l}$ & $20 \mu \mathrm{l}$ & $20 \mu \mathrm{l}$ & $20 \mu \mathrm{l}$ & $40 \mu \mathrm{l}$ \\
\hline $\begin{array}{l}\text { DNA to } \\
\text { was ada } \\
\text { for dige }\end{array}$ & CIP & . & , & & 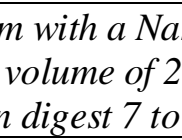 & drop & $\begin{array}{l}\text { Fisher) } \\
1-6, \text { and }\end{array}$ \\
\hline
\end{tabular}

This mixture was incubated at $37^{\circ} \mathrm{C}$ for 1 hour. Afterwards, an additional $1 \mu \mathrm{l}$ of $\mathrm{BamHI}$ and EcoRI was added to digests 1-6. For digest 7, $3 \mu \mathrm{l}$ of BamHI and EcoRI each were added as 
well as $1 \mu$ l of CIP (a phosphatase which dephosphorylates DNA ends to prevent the plasmid that is cut with a single restriction enzyme from ligating with itself). This mixture was allowed to incubate for an additional hour at $37^{\circ} \mathrm{C}$. The digests were then purified with Qiagen Spin Miniprep kit (Qiagen) following the manufacturer's protocol.

\section{Ligation of inserts Into Vector}

The previously prepared mixtures were then subjected to ligation. Six tubes were prepared with $250 \mathrm{ng}$ purified, digested pEX18Tc to which the appropriate digested insert was added at a five-fold molar excess. To this mixture, $3 \mu \mathrm{l}$ of $10 \times$ DNA ligase buffer and $1 \mu \mathrm{l}$ of T4 DNA ligase was added. Sterile $\mathrm{ddH}_{2} \mathrm{O}$ was added to bring the final volume of the ligation mixture to $30 \mu \mathrm{l}$. As controls, mixtures were prepared using only cut pEX18Tc and ligase, omitting the insert, and cut vector alone, both of which were supplemented with the required water to bring the mixtures to $30 \mu \mathrm{l}$. All mixtures were incubated overnight at $16^{\circ} \mathrm{C}$.

\section{Preparation of competent $E$. coli DH5 $\alpha$ cells}

In order to transform the ligations into cells so that the resultant plasmids could be replicated and stored, competent $E$. coli cells were prepared using the following steps:

$400 \mu \mathrm{l}$ of an overnight culture of $E$. coli strain DH5 $\alpha$ was grown in lysogeny broth (LB). This culture was incubated at $37^{\circ} \mathrm{C}$ on a roller drum for 1.5 hours to ensure exponential phase growth.

After 1.5 hours, the tubes of inoculated broth were put on ice for 15 minutes. They were then centrifuged at $14,000 \mathrm{rpm}$ for two minutes at $4^{\circ} \mathrm{C}$. The supernatant was discarded, and the pellet was resuspended using $500 \mu \mathrm{l}$ of ice cold $100 \mathrm{mM} \mathrm{MgCl}_{2}$. This mixture was centrifuged at $14,000 \mathrm{rpm}$ for two additional minutes at $4^{\circ} \mathrm{C}$. The supernatant was discarded, and the pellets were resuspended in $500 \mu \mathrm{l}$ of $100 \mathrm{mM}$ of $\mathrm{CaCl}_{2}$. This mixture was left on ice for 30 minutes 
before the cells were repelleted. The supernatant was discarded. The cells were resuspended in a mixture of $100 \mu \mathrm{l} 15 \%$ glycerol and $100 \mathrm{mM} \mathrm{CaCl}_{2}$ solution. This mixture was stored at $-80^{\circ} \mathrm{C}$ until needed for transformations.

\section{Transformation of ligations into chemically competent $E$. coli}

Chemically competent DH5 $\alpha$ cells were thawed on ice. $100 \mu \mathrm{L}$ of these cells was added to each of 8 tubes containing the eight previously prepared ligation mixes and were vortexed on high for 2-3 seconds. As a positive control, an additional transformation was made using only undigested pEX18Tc plasmid. As a negative control, a transformation mixture was made without a DNA template. These mixtures were incubated on ice for 10 minutes, then at $42^{\circ} \mathrm{C}$ for 1 minute, and back on ice for an additional 10 minutes. $1 \mathrm{ml}$ of sterile LB was added to each transformation, and the cells were then incubated at $37^{\circ} \mathrm{C}$ and approximately $300 \mathrm{rpm}$ for 1 hour to promote expression of the resistance markers on the plasmids. $100 \mu \mathrm{l}$ of this solution was plated on LB plates with Tet(10) (tetracycline, $10 \mu \mathrm{g} / \mathrm{ml}$ ) and spread using glass beads. These 10 agar plates were incubated overnight at $37^{\circ} \mathrm{C}$. Growth was assessed by examining number and size of colonies that formed.

The transformed colonies were transferred onto fresh LB Tet(10) plates using sterile toothpicks and allowed again to grow overnight. In the morning, select colonies (5 from each ligation) were selected for use as templates in colony PCR reactions using the GoTaq green procedure outlined above, substituting the template with select colonies. This was done in order to identify colonies containing insert of the appropriate size. Positive controls were made using K279a gDNA as the template, and a negative control was made substituting $\mathrm{ddH} 2 \mathrm{O}$ for DNA template. 
The results of the PCR were used to identify colonies that contain the transformed plasmid with the correct insert. Promising colonies were subject to plasmid purification (as outlined above, for isolating pEX18Tc originally) to eventually be used in sequencing to confirm that transformation occurred. Additionally, the same colonies were then prepared to conjugate the plasmid from E. coli into S. maltophilia because identical sequence across the entire insert was not deemed to be essential for single crossover insertional disruption of the desired genes (Figure 1).

\section{Conjugation from $E$. coli into $S$. maltophilia}

In order to transfer the plasmid from E. coli into $S$. maltophilia, the helper strain E. coli pRK2013 which allows interspecies conjugation ${ }^{(12)}$ was used. E. coli DH5 $\alpha$ containing the appropriate plasmids were used to inoculate $5 \mathrm{~mL}$ of LB Tet(10). S. maltophilia K279a was placed in $5 \mathrm{ml}$ of LB broth containing no antibiotic. The helper strain of E. coli (pRK2013) was placed in $5 \mathrm{ml}$ of LB broth with kanamycin $(50 \mu \mathrm{g} / \mathrm{ml})$. These were incubated at $37^{\circ} \mathrm{C}$ and approximately 300 rpm overnight.

Following overnight incubation, S. maltophilia K279a was heat shocked at $42^{\circ} \mathrm{C}$ for two hours. $400 \mu \mathrm{l}$ of heat-shocked K279a was mixed with $200 \mu \mathrm{l}$ of donor E. coli (containing the desired pEX18Tc derivative) and $200 \mu \mathrm{l}$ of helper E. coli pRK2013. This mixture was spun at $1500 \mathrm{rpm}$ for 1 minute. The supernatant was discarded and the cells were resuspended in $100 \mu \mathrm{l}$ of plain LB broth. This mixture was place on the mating plates (LB without antibiotics) and incubated overnight at $37^{\circ} \mathrm{C}$.

On the following day, the overnight mating growth was resuspended in $1 \mathrm{ml}$ of drug-free LB broth. $300 \mu \mathrm{l}$ and $100 \mu \mathrm{l}$ of this suspension were plated on agar plates containing Tet 20 and irgasan $25 \mu \mathrm{g} / \mathrm{ml}$. Tetracycline selects for bacteria containing pEX18Tc. Irgasan prevents E. coli 
growth but not $S$. maltophilia growth. Due to an increased generation time because of the high concentrations of antibiotics used and the lower temperature, these plates were incubated for 3 days. Background growth was detected, and so suspected S. maltophilia colonies were isolated using sterile toothpicks and struck for isolation on fresh plates with Tet 20 and irgasan 25 .

Isolated transconjugants were expected to contain an interruption of the target gene. This interruption would occur as a result of the single cross-over event that would occur between the inserted sequence in pEX18Tc and the original insertion sequence on K279a's genome. Interruption would cause any products produced from transcription of the gene to be nonfunctional, as both ends of the gene are incomplete (Figure 1).

To screen for the desired transconjugants, isolated colonies were subject to PCR using GoTaq green procedure outlined above, with an increase in the elongation time (step 4, table 2) to 10 minutes for each cycle. A second PCR was performed with HotStar (with the same increase in the elongation time) because GoTaq Green has limited processivity. For these PCR reactions, the template was colony matter from the isolated S. maltophilia transconjugant colonies. As a positive control K279a genomic DNA was used as template.

A PCR was also performed using the GoTaq green procedure and primers for the sequences on pEX18Tc immediately upstream and downstream of the multicloning locus. A product from this PCR would indicate that there was intact plasmid in the S. maltophilia cells (no insertion into the desired sequence). The normal PCR temperature cycling was used (Table 2), and the gel was run with normal conditions.

\section{Growth of S. maltophilia with $P$. aeruginosa.}

C3719, a cystic fibrosis clinical isolate of $P$. aeruginosa for which the genome sequence is known, was used to assay the growth with the conjugated S. maltophilia colonies. C3719 was 
prepared from freezer stock and struck for isolation. The colonies of both C3719 and each of the transconjugants were inoculated into LB broth and grown overnight at $37^{\circ} \mathrm{C}$. The following morning, $1 \mu \mathrm{l}$ of the inoculated broth was spotted on LB agar plates without antibiotics. Colonies to be tested for effects on growth were placed $1 \mathrm{~cm}$ apart and each pairing was assayed in triplicate. For each of the conjugations, several controls were prepared. One control involved growing two C3719 colonies together, another with C3719 next to wild-type K279a, and finally a third control was prepared by growing the transconjugant $S$. maltophilia colony with wild-type K279a. 


\section{Results}

\section{PCR Optimization}

PCR followed by gel electrophoresis was used to identify the conditions leading to optimal amplification of the desired products. Initially, GoTaq green was used to verify that the primers could be used to amplify the desired gene products (Figure 3).

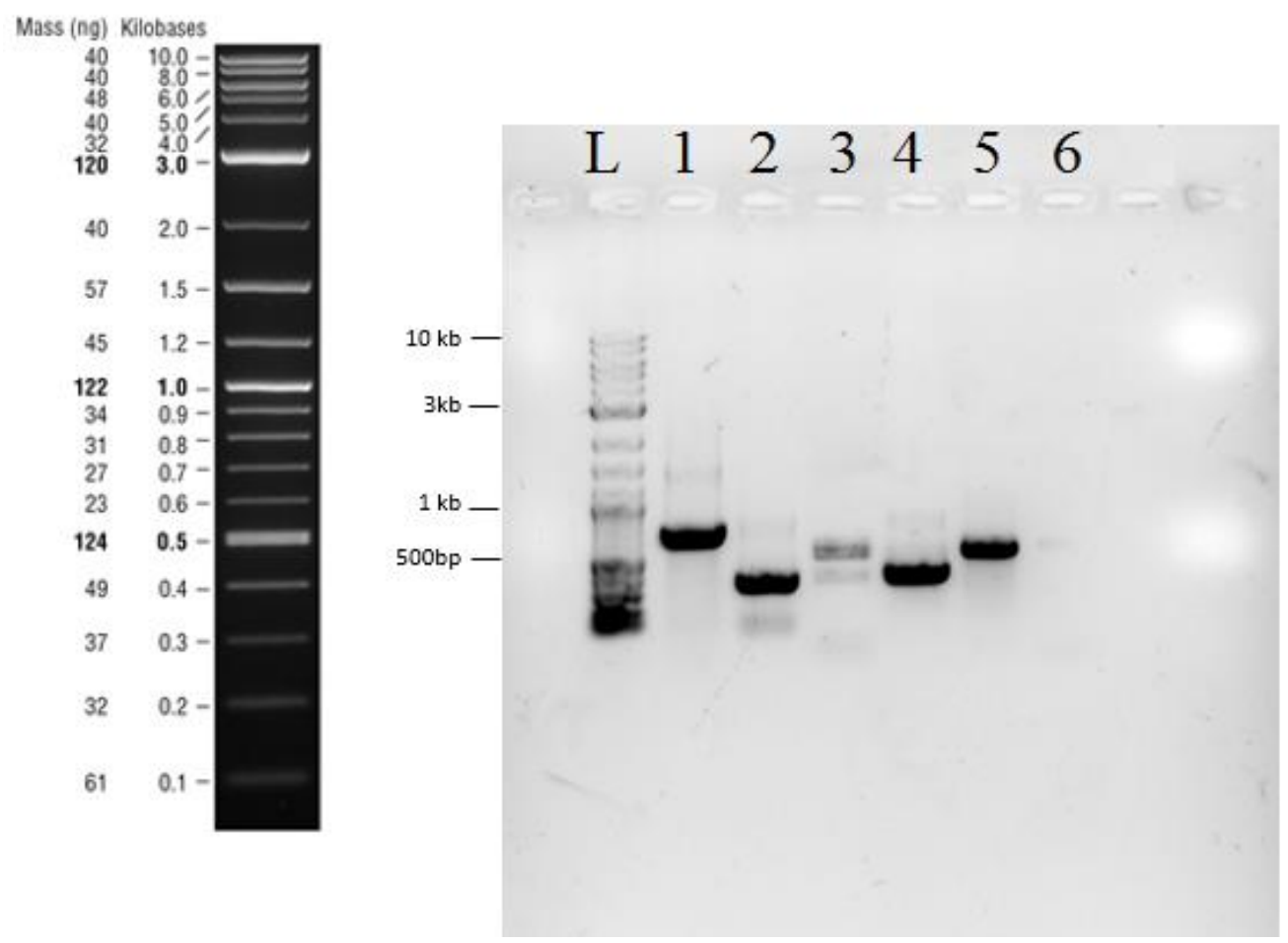

Figure 3: Initial GoTaq Green PCR. L $=2$ Log DNA ladder. PCR1: Smlt_1839, 2: Smlt_3567, 3: Smlt_0278, 4: Smlt_1432, 5: rpfG, 6: Smlt_0184. Desired amplification events appear successful with the given primers and GoTaq Green.

Due to GoTaq green's low fidelity, it was not used for to amplify products for ligation.

Both HotStar and Phusion are described by their manufacturers as having higher fidelity than

GoTaq Green, although their amplification product yield can sometimes be less robust than GoTaq Green. 
Initially, HotStar was only able to successfully amplify Smlt_0184,Smlt_1432, and Smlt_1839 (Figure 4).

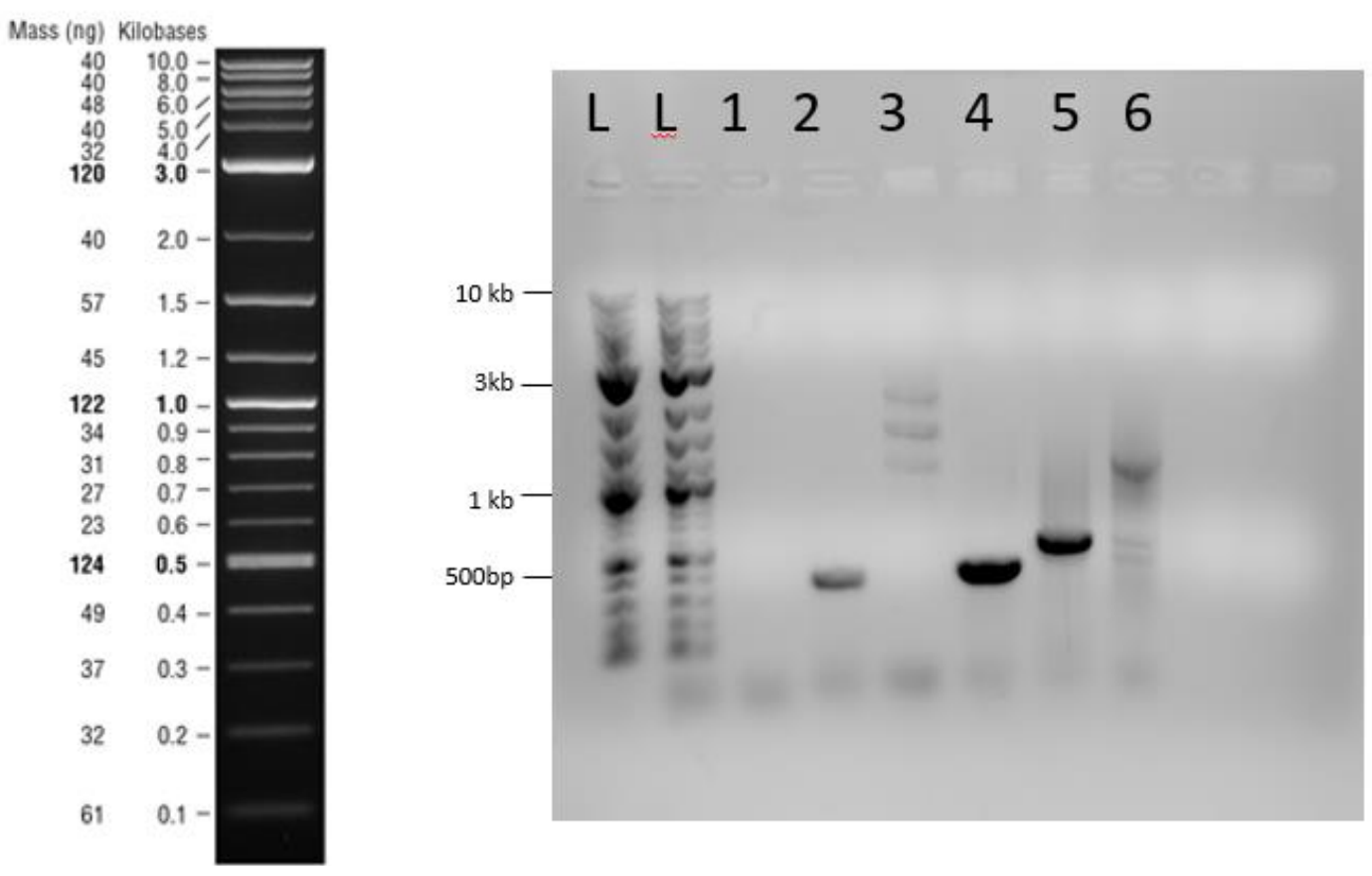

Figure 4: HotStar PCR. L = 2 Log DNA ladder. PCR1: rpfG, 2: Smlt_0184, 3: Smlt_0278, 4: Smlt_1432, 5: Smlt_1839, 6: Smlt_3567. Figure shows successful amplification of Smlt_0184,Smlt_1432, and Smlt_1839 with minimal extra bands. 

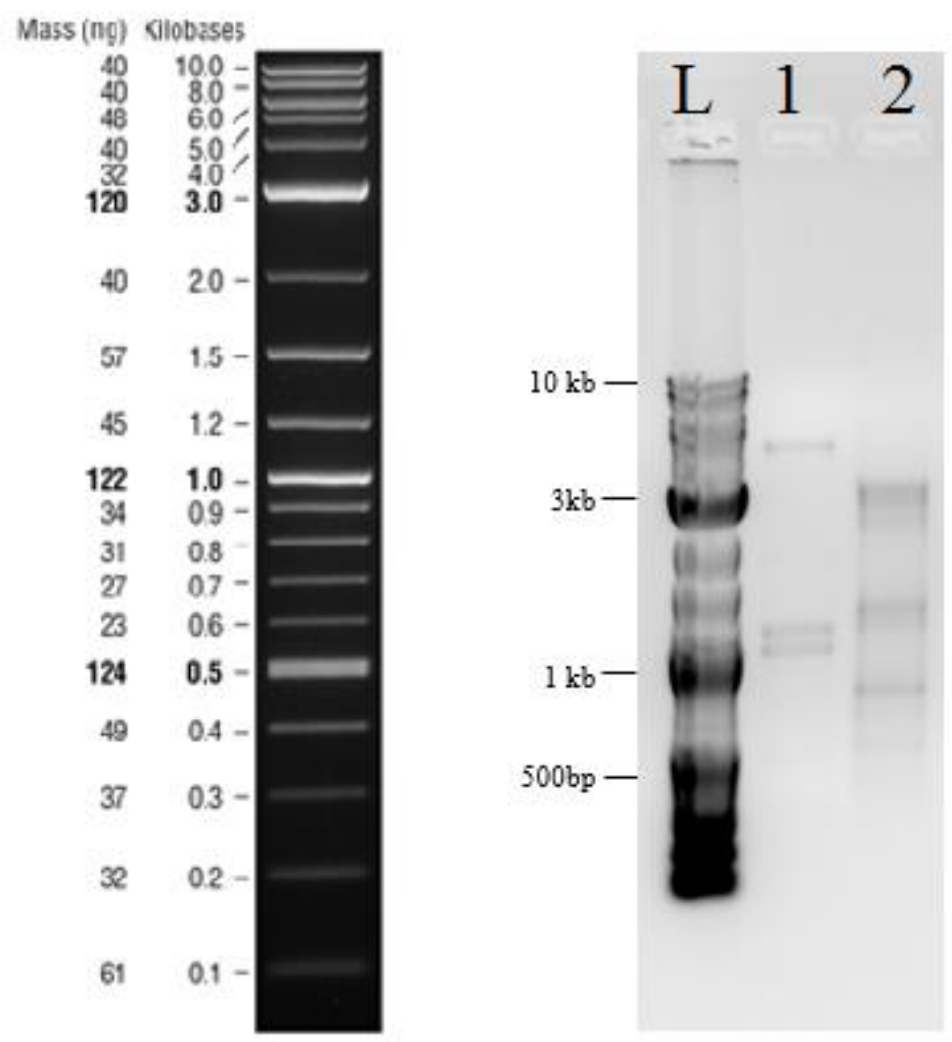

Figure 5: Phusion PCR. L = 2 Log DNA ladder. PCR1: Smlt_0278. PCR 2: Smlt_3567.

Amplification of Smlt_0278 and Smlt_3567 was then attempted with Phusion, but only faint bands (of many sizes in each reaction) were produced (Figure 5). 

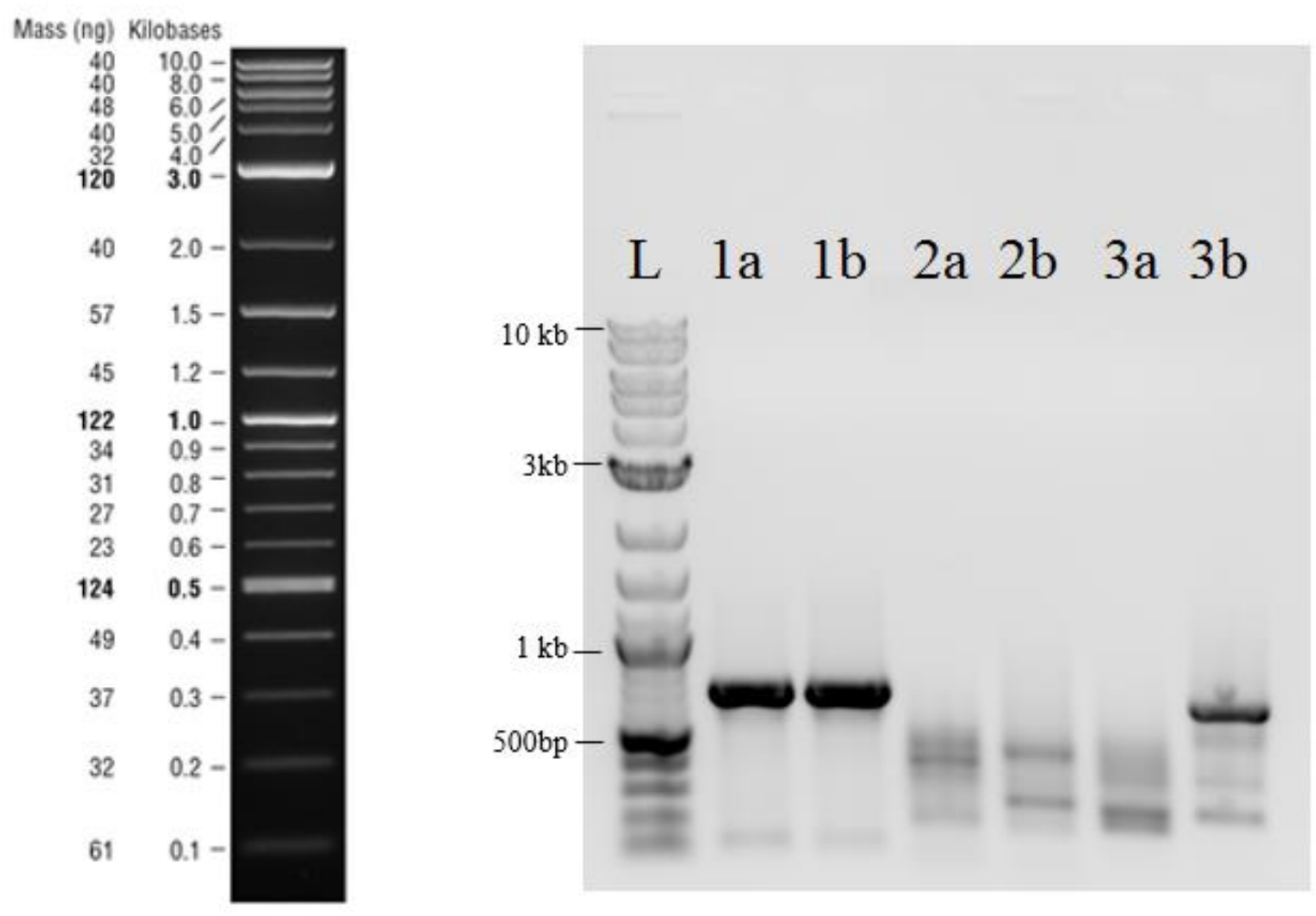

Figure 6: Repeat HotStar PCR using previous PCR mixes as templates. L $=2 \log$ ladder. Reaction 1 corresponds to rpfG. Reaction 2 corresponds to Smlt_0278. Reaction 3 corresponds to Smlt_3567.

Due to the ineffectiveness of Phusion, the amplification of $r p f G, S m l t \_0278$, and Smlt_3567 was repeated with HotStar, using purified PCR products from previous attempts that showed promising results. These reactions resulted in strong bands of $r p f G$ products in both attempts. While reactions with Smlt_0278 and Smlt_3567 produced bands of the desired size, they also produced a significant amount of additional bands of undesired products (Figure 6). 

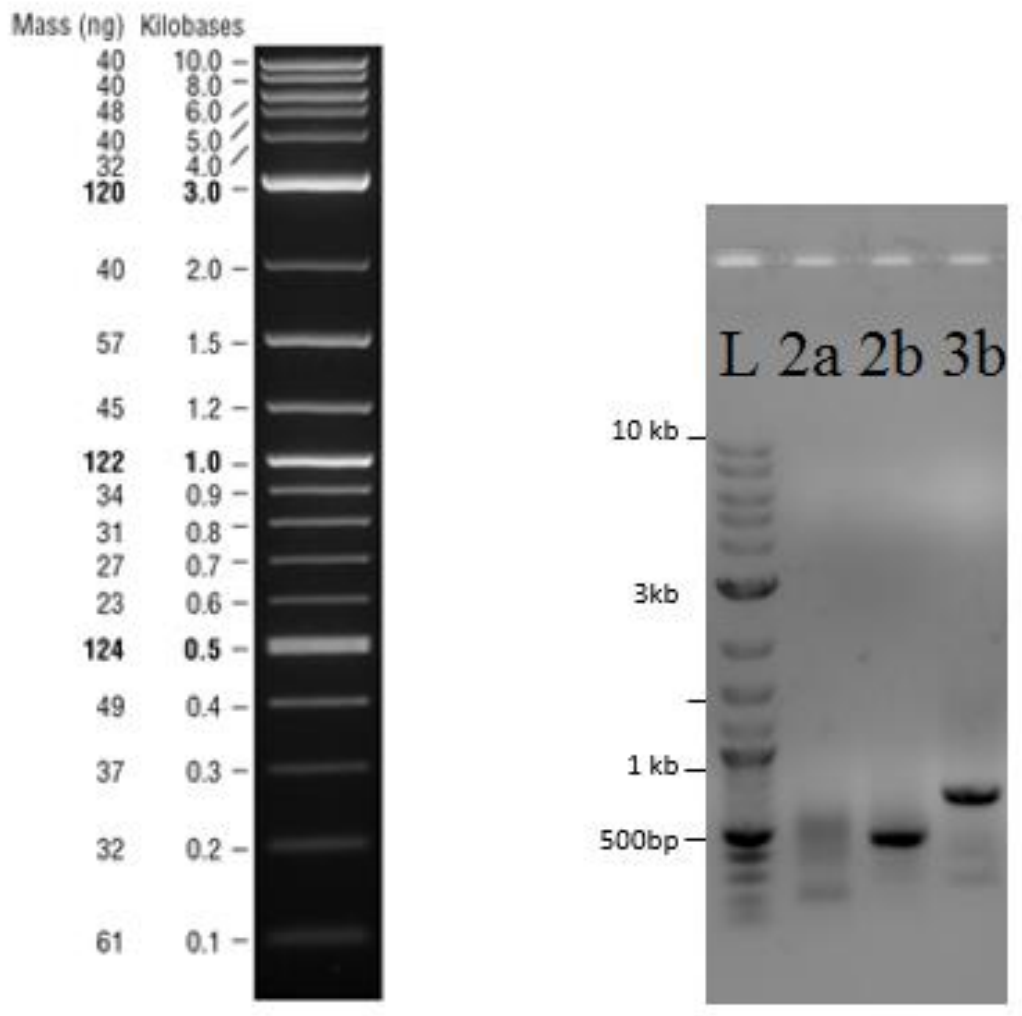

Figure 7: Repeat HotStar PCR using previous PCR mixes as templates. $\mathrm{L}=2 \log$ ladder. Reaction 1 corresponds to $r p f G$. Reaction 2 corresponds to Smlt_0278. Reaction 3 corresponds to Smlt_3567.

Using gel purified products of appropriately sized bands of PCR amplification of Smlt_0278 and Smlt_3567, PCR was repeated with HotStar resulting in successful amplification (Figure 7).

\section{Purifications, Restriction Enzyme Digestion and Ligations}

Once all 6 gene inserts had been amplified, they could be digested in preparation for ligation into the vector. After digestion, the digests were purified and the concentrations of the resultant products were determined to determine the proper volume to achieve 5 fold-molar 
excess of the amplified insert with pEX18 for digestion. Ligations were then performed and prepared for transformation.

\begin{tabular}{|l|l|}
\hline \multicolumn{2}{|l|}{ Table 4: Concentrations of purified digest products } \\
\hline Digest & Concentration $(\mathrm{ng} / \mathrm{nL})$ \\
\hline $1-r p f G$ & 18.9 \\
\hline $2-S m l t \_0184$ & 30.2 \\
\hline $3-$ Smlt_0278 & 28 \\
\hline $4-$ Smlt_1432 & 35.9 \\
\hline $5-$ Smlt_1839 & 37.9 \\
\hline $6-$ Smlt_3657 & 29 \\
\hline $7-$ pEX18Tc & 15.6 \\
\hline
\end{tabular}

\section{Transformation of ligations into chemically competent $E$. coli}

The ligations were then transformed into chemically competent DH5 $\alpha$ cells.

Transformants were plated on LB with tetracycline and incubated. Assessment of the growth is show in Table 5.

\begin{tabular}{|l|l|l|}
\hline \multicolumn{2}{|l|}{ Table 5: Colony densities from plated E. coli on Tet(10) plates post transformation. } \\
\hline Transformation & DNA & CFUs/mL \\
\hline 1 & rpfG & 220 \\
\hline 2 & Smlt_0184 & 1110 \\
\hline 3 & Smlt_0278 & 910 \\
\hline 4 & Smlt_1432 & 2160 \\
\hline 5 & Smlt_1839 & 4920 \\
\hline 6 & Smlt_3567 & 2560 \\
\hline 7 & No insert & 80 \\
\hline 8 & No ligase, no insert & 0 \\
\hline 9 & Positive control: pEX18Tc & Too many to count \\
\hline 10 & No template & 0 \\
\hline
\end{tabular}

Results show that transformation proceeded as expected, as the negative controls (8 and 10) did not show any growth and the remainder did grow as was expected. 
These colonies were transferred onto fresh LB plates and allowed to grow overnight. Five colonies from each ligation examined by colony PCR to identify colonies with insert of the desired size (Figure 8).
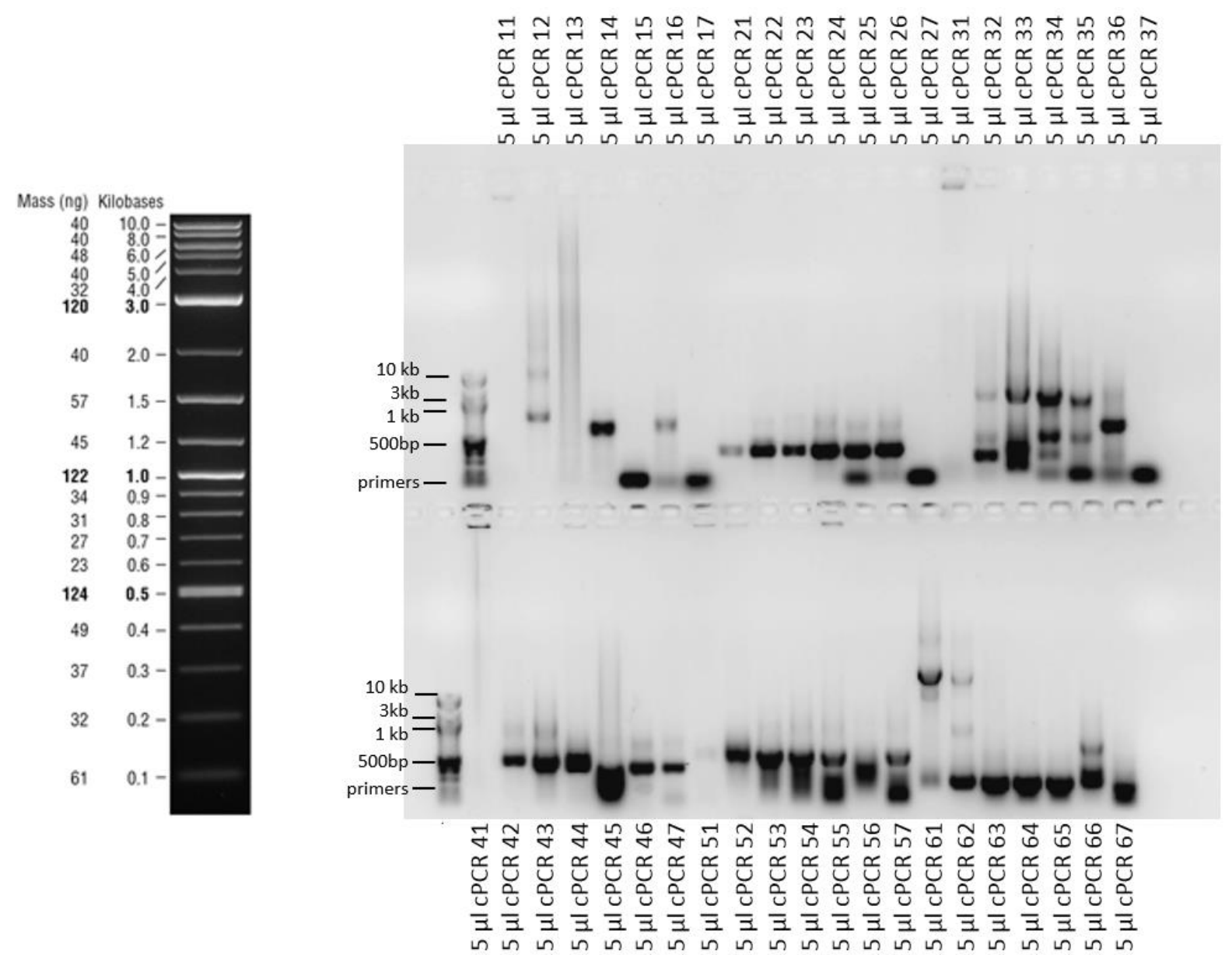

Figure 8: cPCR (colony PCR) of five colonies from each of the 6 transformations. Reactions 11-17 represent colonies transformed with rpfG. 21-27: Smlt_0184. 31-37: Smlt_0278.41-47: Smlt_1432.51-57 Smlt_1839.61-67: Smlt_3567.11-15, 21-25, 31-35, 41-45, 51-55, and 61-65 represent the 5 colony isolates used as templates. Reactions 16, 26, 36, 46, 56, and 66 represent positive controls for each reaction where the template was $1 \mu$ l of K279a DNA. Reactions 17, 27, 37, 47, 57, and 67 were negative controls, where $1 \mu \mathrm{lddH_{2 }} \mathrm{O}$ was used as a template. The negative controls show bands corresponding to the primers that were added. 

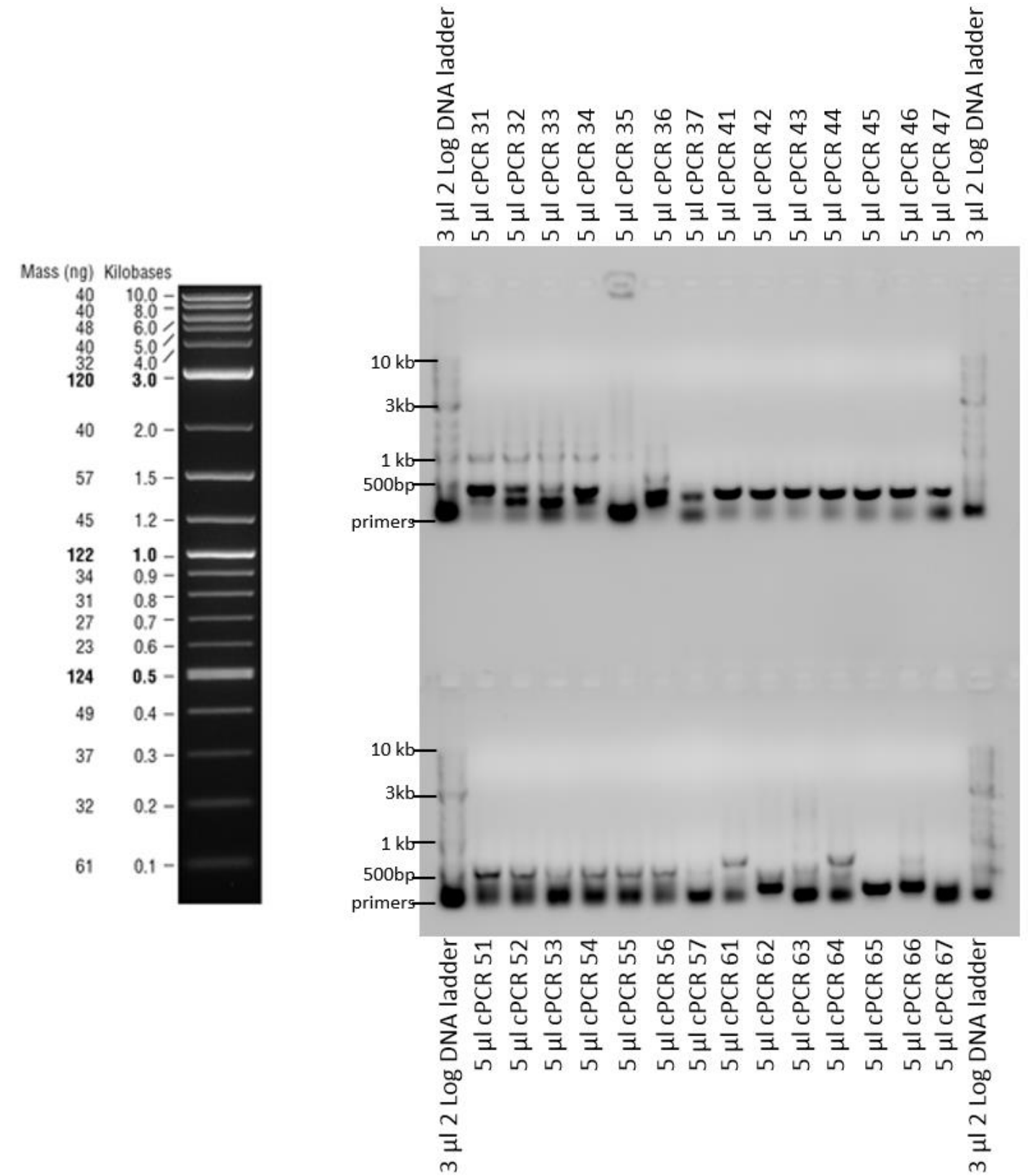

Figure 9: Repeat cPCR (colony PCR) of five colonies from 4 of the transformations. Reactions 31-37 represent Smlt_0278.41-47: Smlt_1432.51-57 Smlt_1839.61-67: Smlt_3567.31-35, 41-45, 51-55, and 61-65 represent the 5 colony isolates used as templates. Reactions 36, 46, 56, and 66 represent positive controls for each reaction where the template was $1 \mu \mathrm{l}$ of K279a DNA. Reactions 37, 47, 57, and 67 were negative controls, where $1 \mu \mathrm{lddH} 2 \mathrm{O}$ was used as a template. The negative controls show bands corresponding to the primers that were added. 
Because PCR on colonies from transformations 3-6 failed to identify colonies producing a single band of the desired size, the PCRs were repeated using different colonies (Figure 9).These PCR reactions (Figure 8 and 9) identified for two rpfG, two Smlt_0184, four Smlt_0278, three Smlt_1432, two Smlt_1839, and two Smlt_3657 colonies that produced bands that appeared to be the appropriate size. Plasmids from these colonies were then purified and used as templates in subsequent PCR reactions (Figure 10).
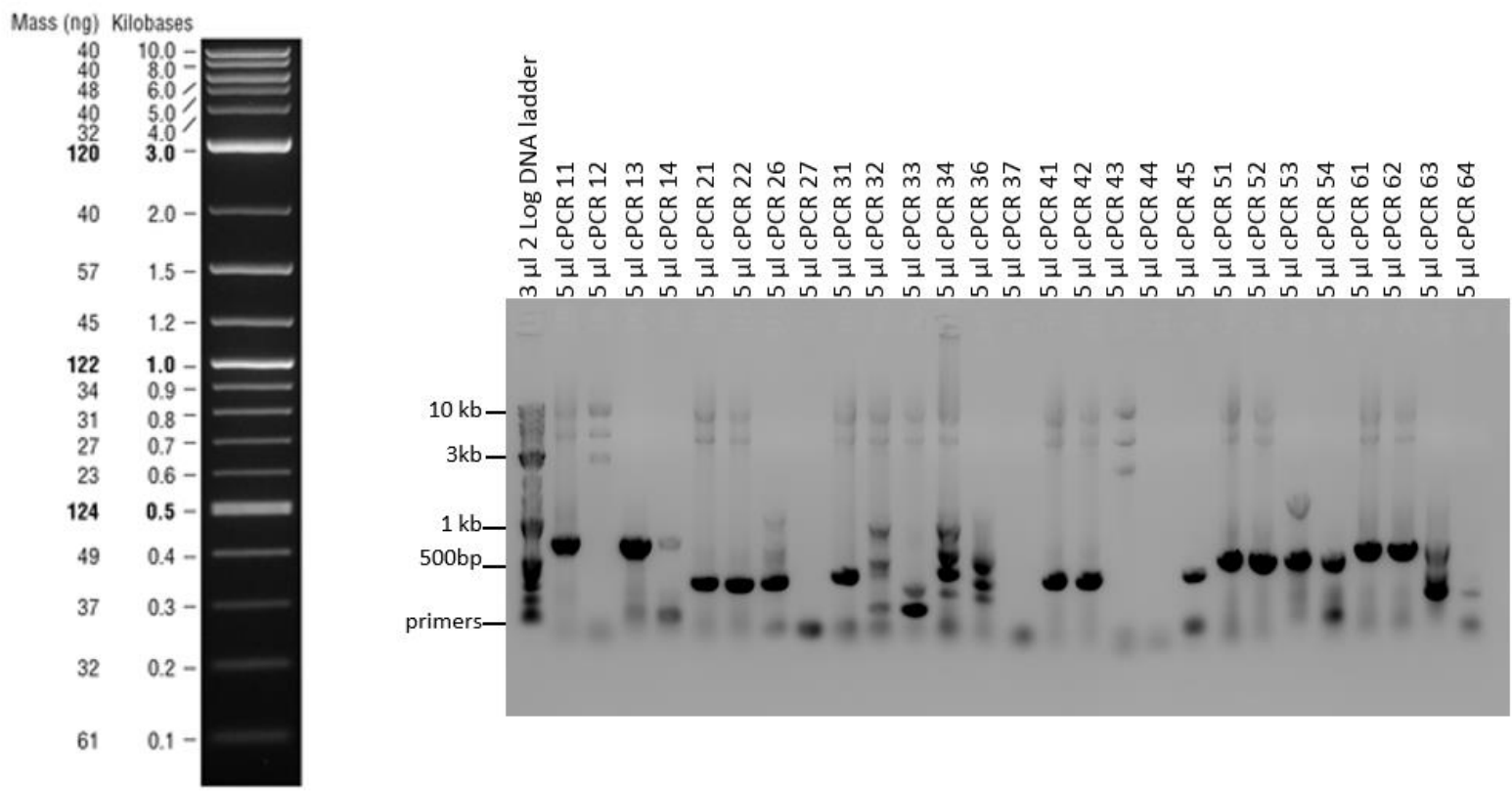

Figure 10: cPCR post plasmid purification. Reactions 11-14 represent $r p f G .21,22$, 26, 27: Smlt_0184.31-34: Smlt_0278.41-45: Smlt_1432.51-54: Smlt_1839.6164: Smlt_3657. 13, 26, 33, 44, 53, and 63 represent positive controls (pEX18Tc used as a template). 14, 27, 34, 45, 54, and 64 represent negative controls, where $\mathrm{ddH} 2 \mathrm{O}$ was used as a template. The negative controls show bands corresponding to the primers that were added.

These results identified the best candidates to be used for conjugation into S. maltophilia $(11,21,22,41,42,51,52,61$, and 62). There were no reactions for Smlt_0278 that produced 
results sufficient to proceed with conjugation (additional bands, bands in the negative control), so that reaction was left out for the remainder of experimentation.

\section{Conjugation from $E$. coli into $S$. maltophilia}

The previously identified nine colonies were used for conjugation. All of the conjugations into $S$. maltophilia had at least 30 colonies after 2 days incubation at room temperature. After 3 days there was background growth on all of the plates, so larger colonies were transferred onto fresh LB Tet (20) Irg (25) plates using sterile toothpicks and grown

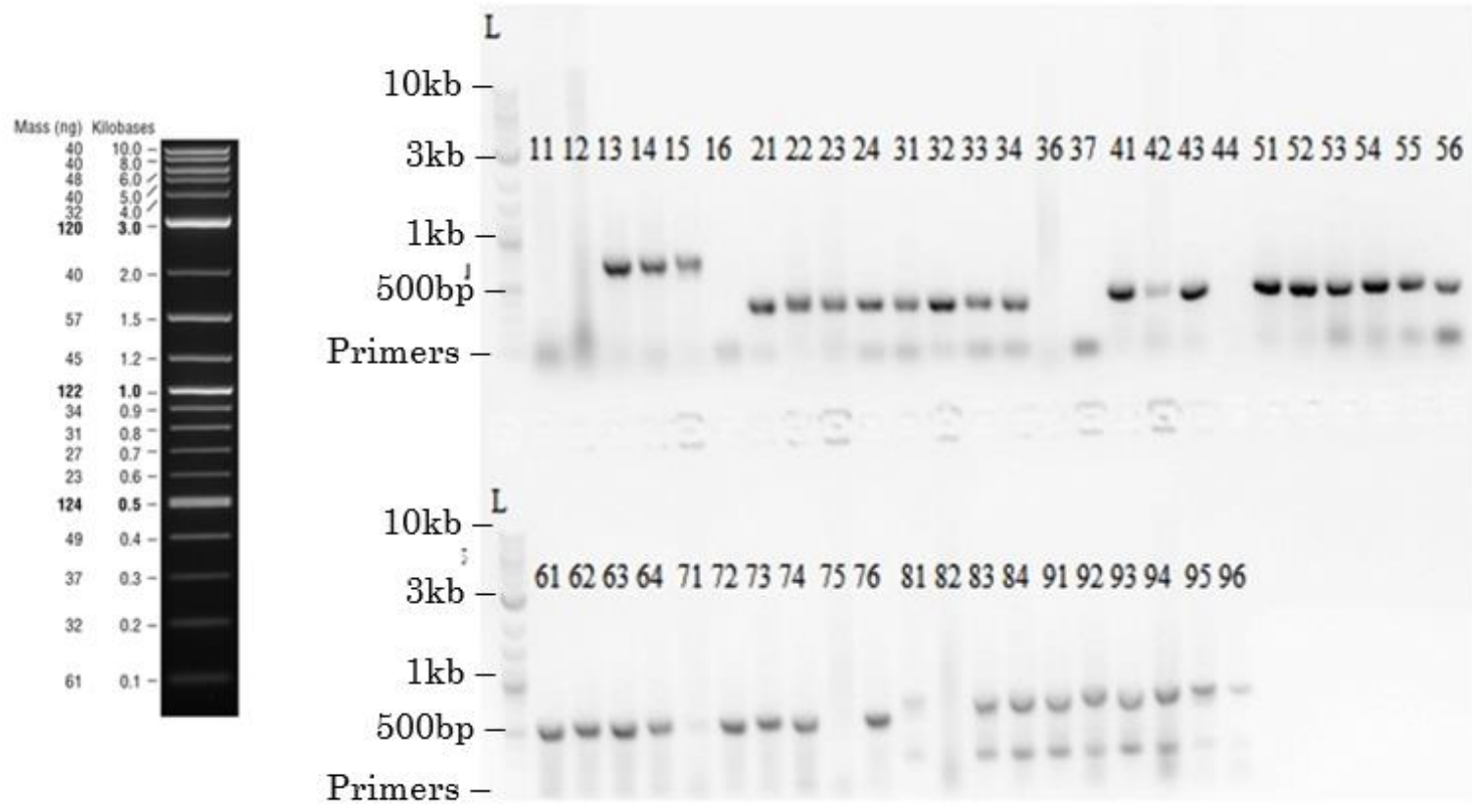

Figure 11: cPCR of isolate $S$. maltophilia colonies after conjugation. L corresponds to two log ladder used for size identification. Reaction 11-14 are four colonies for the first conjugation rpfG. 21-24: conjugation 2, 31-34 conjugation 3, both corresponding to Smlt_0184.41-44: conjugation 4, 51-54: conjugation 5, both corresponding to Smlt_1432.61-64: conjugation 6, 71-74: conjugation 7, both corresponding to Smlt_1839.81-84: conjugation 8, 91-94: conjugation 9, both corresponding to Smlt_3657. 15, 35, 55, 75, and 95 correspond to positive controls in which wild-type K279a was added in place of transconjugant colonies. 16, 36, 56, 76, and 96 correspond to controls in which $\mathrm{ddH} 2 \mathrm{O}$ was added as a template. 
overnight at $37^{\circ} \mathrm{C}$. These were then streaked for isolation and used in another PCR to assay for desired products (Figure 11).

The products after conjugation were expected to include bands of the original insert size as well as bands approximately $6 \mathrm{~kb}$ larger than the original genes because they should include the entire plasmid integrated into the gene (Figure 1). Findings depicted in Figure 11 suggest that conjugation may not have occurred in the desired location, but this is not entirely conclusive because GoTaq Green often is not processive enough to amplify more than $2000 \mathrm{bp}$.

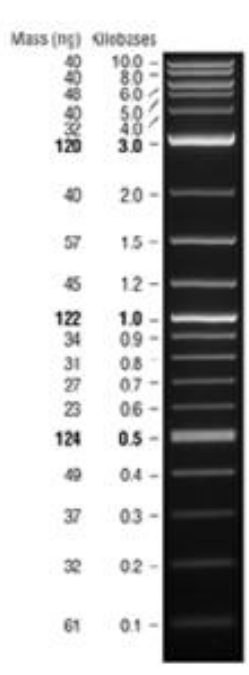

\section{$\begin{array}{lllllllllllll}\mathrm{L} & 1 \mathrm{a} & 1 \mathrm{~b} & 2 & 3 & 4 & 5 & 6 & 7 & 8 & 9 & 10 & 11\end{array}$}
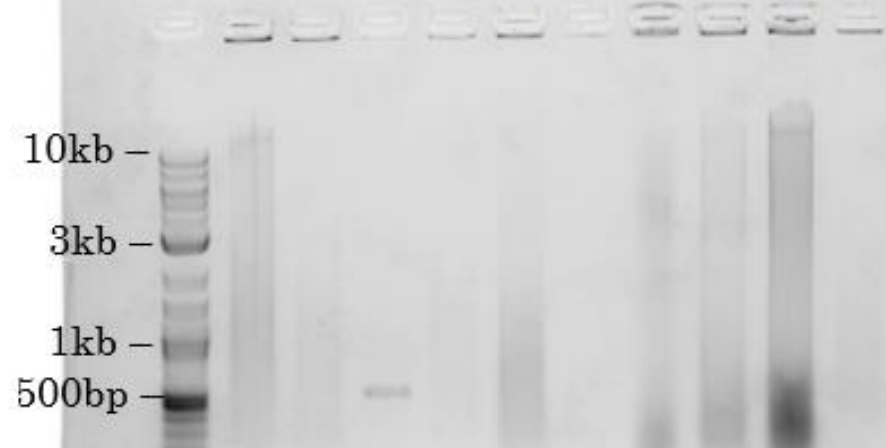

Figure 12: Repeat cPCR of transconjugant colonies using pEX18Tc primers. $\mathrm{L}=\log$ 2 ladder for size comparison. Conjugation $1(r p f G)$ was plated in lanes $1 \mathrm{a}$ and $1 \mathrm{~b}$, with two different isolated colonies. Lane 2 and 3 are colonies from conjugations 2 and 3 (Smlt_0184).4/5: Conjugations 4 and 5 (Smlt_1432).6/7: Conjugations 6 and 7 (Smlt_1839). 8/9: Conjugations 8 and 9 (Smlt_3657).

The PCR was repeated with only 2 colonies representing each gene to be interrupted with primers for pEX18Tc (Figure 12). These PCR reactions should show a product only when the plasmid is present without recombination at the gene of interest. If the cloned insert is intact the PCR will give a product that is the size of the insert as the primers will amplify across the insert. 
If the insert has recombined into the chromosome through single crossover, the primers will be pointing out and would lead to amplification of the entire genome except the plasmid sequence to produce a band, and this is extremely unlikely to happen in the extension time allotted. The 9 transconjugant colonies were renamed (Table 6). K279a $\Delta r p f G \# 1, \mathrm{~K} 279 \mathrm{a} \Delta$ smlt0184\#2, K279a $\Delta$ smlt1432\#2, K279a $\Delta$ smlt1839\#1, and K279a $\Delta$ smlt3657\#2 lacked bands the size of the inserts and were assumed to represent the appropriate construct.

\begin{tabular}{|l|l|l|l|}
\hline \multicolumn{4}{|l|}{ Table 6: New naming scheme for transconjugant colonies. } \\
\hline Insert & Conjugation & Original isolate colony \# & New name \\
\hline$r p f G$ & 1 & 11 & K279a $\Delta$ rpfG\#1 \\
\hline$r p f G$ & 1 & 12 & K279a $\Delta$ rpfG\#2 \\
\hline Smlt_0184 & 2 & 21 & K279a $\Delta$ smlt0184\#1 \\
\hline Smlt_0184 & 3 & 41 & K279a $\Delta$ smlt0184\#2 \\
\hline Smlt_1432 & 4 & 1 & K279a $\Delta$ smlt1432\#1 \\
\hline Sml__1432 & 5 & 21 & K279a $\Delta$ smlt1432\#2 \\
\hline Smlt_1839 & 6 & 41 & K279a $\Delta$ smlt1839\#1 \\
\hline Smlt_1839 & 7 & 1 & K279a $\Delta$ smlt1839\#2 \\
\hline Smlt_3657 & 8 & 23 & K279a $\Delta$ smlt3657\#1 \\
\hline Smlt_3657 & 9 & 41 & K279a $\Delta$ smlt3657\#2 \\
\hline
\end{tabular}

\section{Growth of S. maltophilia with P. aeruginosa}

Co-growth of $P$. aeruginosa and one isolate of each knockout strain was assayed.

Transconjugants were chosen and individually plated $1 \mathrm{~cm}$ away from $P$. aeruginosa $\mathrm{C} 3719$ in triplicate. The plates were incubated overnight at $37^{\circ} \mathrm{C}$. Growth was examined for qualitative changes in compared to P. aeruginosa and wild type K279a (Figure 13). There were little to no changes. 

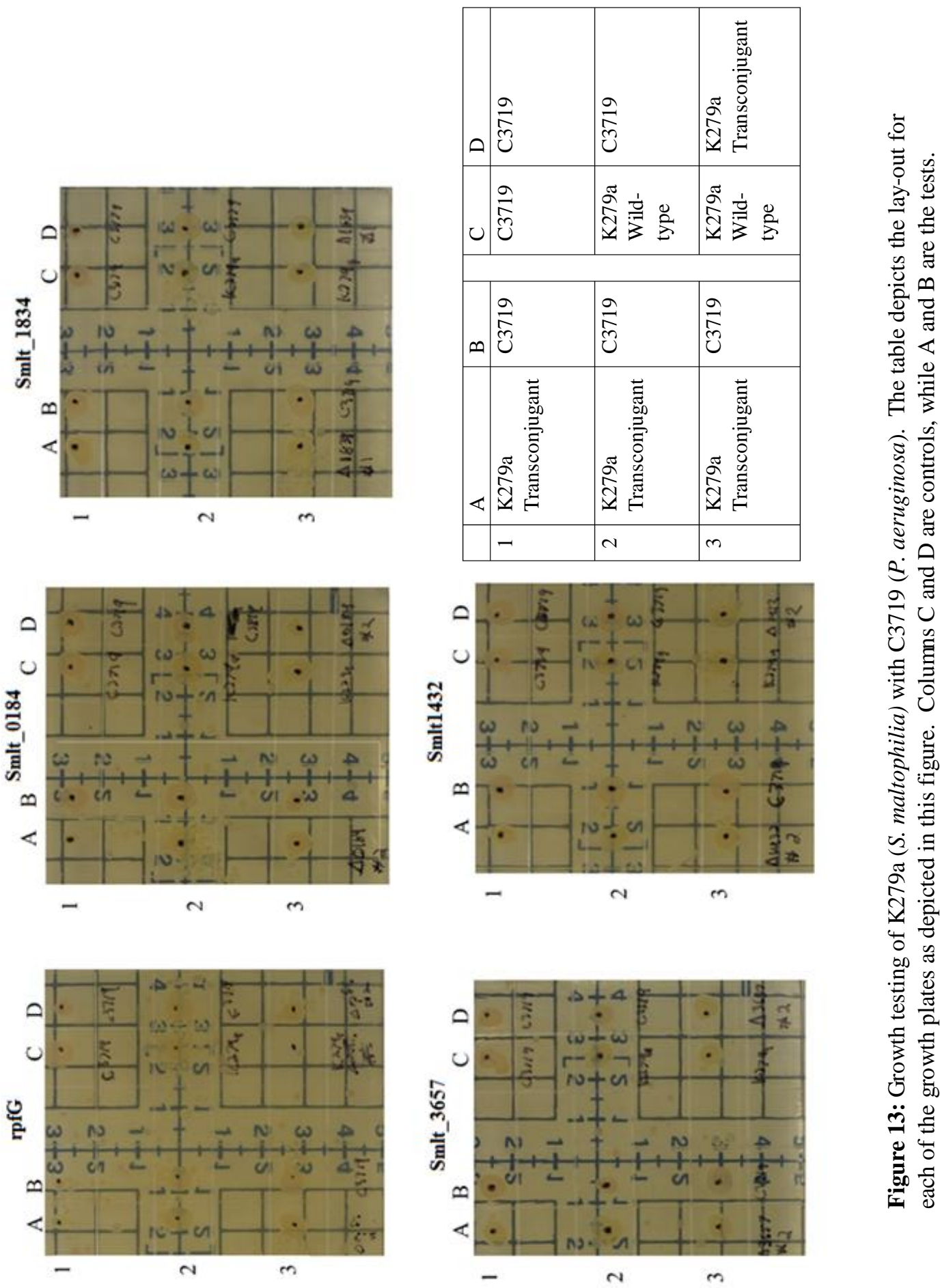

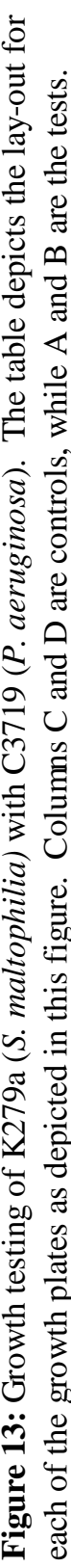




\section{Discussion}

\section{PCR optimization}

Out of the three polymerases examined for amplification of the desired gene products (GoTaq Green, HotStar, and Phusion), HotStar appeared to be the best choice due to the balance of product yield and polymerase fidelity. It produced consistent results, although it occasionally required gel purification to isolate the desired amplification products.

GoTaq green was a useful polymerase, as it was an efficient means of running PCRs (low cost, less complicated mix) and it consistently showed bands of the desired size. For amplifications resulting in products to be used for cloning it was not used due to its low fidelity. However, it was used extensively to determine whether constructs had the appropriate size.

\section{Transformation of Ligations into Chemically Competent $E$. coli}

E. coli DH5 $\alpha$ was chosen as the initial species for transformation as it provided an easy, efficient way to replicate the intact plasmid. The transformed colonies that were produced were easy to handle; they could be frozen (for long term storage) and readily grew on simple LB agar plates and in liquid broths as needed. A large stock was easily produced and then used for conjugation.

The results of the PCRs for transformed colonies of E. coli (Figure 8, Figure 9, and Figure 10) only occasionally showed evidence of successful transformation of the ligated plasmid. In many cases, there were additional unexpected products. Explanations for this phenomenon include insertion of multiple copies of the cloned sequence into the plasmid in tandem or nonspecific binding of the primers to E. coli genomic DNA. As a result of the additional bands, several attempts were required to find colonies that contained the desired 
inserts. Successful insertion of Smlt_0278 was not identified after multiple screening attempts, so this gene was not included in conjugations and growth assays

\section{Conjugation and Growth testing}

Cloning produced plasmids in E. coli that appear to have the desired sequence for five of the desired genes. These plasmids were conjugated into S. maltophilia K279a resulting in transconjugants with the desired antibiotic resistances from each plasmid. However, there appeared to be conflicting evidence as to whether or not the conjugation and recombination occurred as intended. pEX18Tc lacks a S. maltophilia origin of replication and is therefore unable to replicate ${ }^{(13)}$. Additionally, without the tetracycline resistance gene from pEX18Tc plasmid, the K279a strain of S. maltophilia cannot grow on tetracycline present on the selective plates. E. coli cannot grow in the presence of irgasan. This means that only S. maltophilia that has acquired tetracycline resistance (most likely from the plasmid) and incorporated the associated genes into its chromosome can grow on the irgasan and Tetracycline. Furthermore, the colonies appeared in coloration more like S. maltophilia than E. coli leading to the assumption that they are not $E$. coli colonies that acquired irgasan resistance from S. maltophilia.

The growth of $S$. maltophilia colonies on the irgasan and Tetracycline plates is evidence that the transformation and recombination was successful. However, two rounds of PCR (with GoTaq Green and HotStar) did not show strong evidence of recombination events. Due to the interruption of the gene, the PCR should result in bands of two different sizes: one the size of the gene and the other $6.2 \mathrm{kB}$ larger (Figure 1). Instead, the products of amplification, for the most part, were the same size as the original gene.

One explanation for this event could be that the recombination did not result in the gene being interrupted. This could occur if there was another region on $\mathrm{pEX} 18 \mathrm{Tc}$ that contained a 
similarity the K279a genome for which recombination could occur. In this case, the plasmid would recombine and confer tetracycline resistance to the bacteria without knocking out the gene, and the resulting PCR would only amplify the original gene.

It is possible that all the colonies we isolated were spontaneous tetracycline mutants, but the probability of that happening is quite small (and the negative controls did not show any growth). It is also possible that unlike our expectations pEX18Tc is able to replicate in $S$. maltophilia. The actual requirements for replication in S. maltophilia are unknown, but pEX18Tc is only known to contain an origin of replication for E. coli, and S. maltophilia is much more closely evolutionarily related to $P$. aeruginosa, where pEX18Tc cannot replicate, than it is to E. coli. Recent unpublished work in the lab of Dr. Deborah Yoder-Himes suggests that pEX18Tc is unable to replicate in K279a. The most likely explanation, however, is that the smaller PCR product is preferentially made as large products can be used as templates for small products in subsequent cycles but small products cannot be templates to amplify the large product (Figure 1). As such, this PCR was an inconclusive test of the structure of the transconjugants.

Even if the conjugation and recombination resulted in the desired construct and the gene was interrupted, significant difference in the growth of $S$. maltophilia in the presence of $P$. aeruginosa was not observed in any of the trials. Direct side-by-side growth showed no difference from the control (Figure 13). This finding suggests that the insertional interruption had no effects on growth in this in vitro setting. However, this may or may not relate to how these transconjugants would behave in vivo settings. Significant signaling may occur when the two bacteria grow together, or only in models that place the two species in similar environments as found in the human respiratory tract. Further study could be done to determine if any of these 
transconjugants have significant effect on growth in models that promote biofilm formation and settings that mimic the environment found in cystic fibrosis patients. Additionally, it is possible that $1 \mathrm{~cm}$ is too far apart for effectors released by one of these species to impact growth of the other species. Bacteria are in much closer proximity in biofilm habitats.

In conclusion, it is uncertain at this time if insertional interruption was successful. However, the E. coli transformants have shown that they have successfully taken up plasmids with the desired inserts. The purified plasmids from these are awaiting sequencing at this time. Experiments proceeded without sequencing of the inserts because, since they would be used to interrupt the respective gene, $100 \%$ identity, while useful, was not deemed to be essential. Assuming sequencing confirms the correct insertion, these colonies can still be used to reattempt insertion interruption with $S$. maltophilia and then subsequent growth testing with $P$. aeruginosa. Additionally, more models of growth that more accurately mimic the biofilm environment created between these two species in cystic fibrosis patients could be tested with both current existing and new transconjugant strains of $S$. maltophilia. Finally, it is possible that additional signaling receptors have yet to be identified that play the most significant role in the growth interplay between $S$. maltophilia and $P$. aeruginosa in biofilms in the cystic fibrosis lung. 


\section{References}

1. Ciofu, O., Hansen, C., \& Hoiby, N. (2013). Respiratory Bacterial Infections in Cystic Fibrosis. Current Opinion in Pulmonary Medicine Curr Opin Pulm Med, 13(3), 251-258. doi:10.1097/MCP.0b013e32835fafc

2. Goncalves-Vidigal, P., Grosse-Onnebrink, J., Mellies, U., Buer, J., Rath, P., \& Steinmann, J. (2011).

Stenotrophomonas maltophilia in cystic fibrosis: Improved detection by the use of selective agar and evaluation of antimicrobial resistance. Journal of Cystic Fibrosis, 10(6), 422-427. doi:10.1016/j.jcf.2011.06.010

3. Looney, W., Narita, M., \& Mühlemann, K. (2009). Stenotrophomonas maltophilia: An emerging opportunist human pathogen. The Lancet Infectious Diseases, 9(5), 312-323.

doi:10.1016/S1473-3099(09)70083-0

4. Pompilio, A., Crocetta, V., Confalone, P., Nicoletti, M., Petrucca, A., Guarnieri, S., . . Bonaventura, G. (2010). Adhesion to and biofilm formation on IB3-1 bronchial cells by

Stenotrophomonas maltophilia isolates from cystic fibrosis patients. BMC Microbiology

BMC Microbiol, 10, 102-116. doi:10.1186/1471-2180-10-102

5. Pompilio, A., Pomponio, S., Crocetta, V., Gherardi, G., Verginelli, F., Fiscarelli, E., . . Bonaventura, G. (2011). Phenotypic and genotypic characterization of Stenotrophomonas maltophilia isolates from patients with cystic

fibrosis: Genome diversity, biofilm formation, and virulence. BMC Microbiology BMC Microbiol, 11(1), 159-175. doi:10.1186/1471-2180-11-159

6. Pompilio, A., Ciavardelli, D., Crocetta, V., Consalvo, A., Zappacosta, R., Ilio, C., \& Bonaventura, G. (2014). Stenotrophomonas maltophilia Virulence and Specific

Variations in Trace Elements during Acute Lung Infection: Implications in Cystic

Fibrosis. PLoS ONE, 9(2), 1-17. doi:10.1371/journal.pone.0088769

7. Rudkjøbing, V., Thomsen, T., Alhede, M., Kragh, K., Nielsen, P., Johansen, U., . . Bjarnsholt, T. (2012). The microorganisms in chronically infected end-stage and non-end-stage cystic fibrosis patients. FEMS Immunology \& Medical Microbiology FEMS Immunol Med Microbiol, 65(2), 236-244. doi:10.1111/j.1574-695X.2011.00925.x

8. Samonis, G., Karageorgopoulos, D., Maraki, S., Levis, P., Dimopoulou, D.,

Spernovasilis, N., ... Falagas, M. (2012). Stenotrophomonas maltophilia Infections in a

General Hospital: Patient Characteristics, Antimicrobial Susceptibility, and Treatment

Outcome. PLoS ONE, 7(5), 1-7. doi:10.1371/journal.pone.0037375

9. Tümmler, B., \& Kiewitz, C. (1999). Cystic fibrosis: An inherited susceptibility to bacterial respiratory infections. Molecular Medicine Today, 5(8), 351-358.

10. Vrankrijker, A., Wolfs, T., \& Ent, C. (2010). Challenging and emerging pathogens in cystic fibrosis. Paediatric Respiratory Reviews, 11(4), 246-254.

doi:10.1016/j.prrv.2010.07.003

11. Crossman, L., Gould, V., Dow, J., Vernikos, G., . . (2008). The complete genome, comparative and functional analysis of Stenotrophomonas maltophilia reveals an organism heavily shielded by drug resistance determinants. Genome Biology, 9:R74. doi:10.1186/gb-2008-9-4r74

12. Ditta, G., Stanfield, S., Corbin, D., Helinski, D. (1980). Broad host range DNA cloning system for Gramnegative bacteria: Construction of a gene bank of Rhizobium meliti. Proceedings of the National Academy of Sciences of the United States of America 77(12), 7347-7351.

13. Hoang T.T., Karkhoff-Schweizer R.R., Kutchma A.J., Schweizer H.P.. (1998) A broad-host-range Flp-FRT recombination system for site-specific excision of chromosomally-located DNA sequences: application for isolation of unmarked Pseudomonas aeruginosa mutants. Gene 28;212(1): 77-8

14. Das, M., Sandhu, P., Gupta, P., Rudrapaul, P. ... (2016) "Attenuation of Pseudomonas aeruginosa biofilm formation by Vitexin: A combinatorial study with azithromycin and gentamicin." Scientific Reports 6, Article number: 23347

15. Lasarre, B., and M. J. Federle. "Exploiting Quorum Sensing To Confuse Bacterial Pathogens." Microbiology and Molecular Biology Reviews 77.1 (2013): 73-111.

16. Kievit, T. R. De. "Quorum Sensing in Pseudomonas Aeruginosa Biofilms."Environmental Microbiology 11.2 (2009): 279-88. 